\title{
ON THE EXPECTATION OF TOTAL DISCOUNTED OPERATING COSTS UP TO DEFAULT AND ITS APPLICATIONS
}

\author{
JUN CAI, ${ }^{* * *}$ University of Waterloo \\ RUNHUAN FENG, ${ }^{* * *}$ University of Wisconsin-Milwaukee \\ GORDON E. WILLMOT, ${ }^{*}$ University of Waterloo
}

\begin{abstract}
In this paper we first consider the expectation of the total discounted claim costs up to the time of ruin, and then, more generally, we study the expectation of the total discounted operating costs up to the time of default, which is the first passage time of a surplus process downcrossing a given level. These two quantities include the expected discounted penalty function at ruin or the Gerber-Shiu function, the expected total discounted dividends up to ruin, and other interesting quantities as special cases among a class of risk processes. As an illustration, we consider a piecewise-deterministic compound Poisson risk model. This model recovers many risk models appearing in the literature such as the compound Poisson risk models with interest, absolute ruin, dividends, multiple thresholds, and their dual models. We derive and solve the integro-differential equation for the expected present value of the total discounted operating costs up to default. The solutions to the expected present value of the total discounted operating costs up to default can be used as a unified approach to solving many ruin-related quantities. As applications, we derive explicit solutions for the expected accumulated utility up to ruin, the absolute ruin probability with varying borrowing rates, the expected total discounted claim costs up to ruin, the Gerber-Shiu function with two-sided jumps, and the price for a perpetual American put option with two-sided jumps.
\end{abstract}

Keywords: Claim cost; operating cost; time of ruin; time of absolute ruin; time of default; dividend; dividend threshold; dividend barrier; utility; piecewise-deterministic compound Poisson process; defective renewal equation; compound geometric distribution

2000 Mathematics Subject Classification: Primary 91B30

Secondary 91B70; 91B28

\section{Introduction}

Let $\left\{X_{t}, t \geq 0\right\}$ be a surplus process for an insurance company with initial surplus $X_{0}=x$ under measure $\mathrm{P}^{x}$, let $\tau=\inf \left\{t: X_{t}<0\right\}$ be the time of ruin, and let $\delta \geq 0$ be a constant representing the force of interest. Recently, the following two functions have attracted a lot of interest in the literature. One is the expected discounted penalty at ruin, also called the Gerber-Shiu function, denoted by

$$
m(x)=\mathrm{E}^{x}\left[\mathrm{e}^{-\delta \tau} w\left(X_{\tau-},\left|X_{\tau}\right|\right) \mathbf{1}(\tau<\infty)\right],
$$

Received 5 February 2009; revision received 29 April 2009.

* Postal address: Department of Statistics and Actuarial Science, University of Waterloo, Waterloo, Ontario N2L 3G1, Canada.

** Email address: jcai@math.uwaterloo.ca

*** Postal address: Department of Mathematical Sciences, University of Wisconsin-Milwaukee, PO Box 413, Milwaukee, WI 53202-0413, USA. 
where the expectation is taken under the measure $\mathrm{P}^{x}, w(x, y)$ is a function defined on $[0, \infty) \times$ $(0, \infty)$ representing penalty at ruin, and $\mathbf{1}(A)$ is the indicator of an event $A$, namely, $\mathbf{1}(A)=1$ if $A$ is true and $\mathbf{1}(A)=0$ if $A$ is false. The other function is the expected total discounted dividends up to ruin defined by

$$
V(x)=\mathrm{E}^{x}\left[\int_{0}^{\tau} \mathrm{e}^{-\delta t} \mathrm{~d} D(t)\right]
$$

where $D(t)$ denotes the accumulated dividend paid up to time $t$. Details on applications of these two quantities can be found in many papers, among which are Gerber and Shiu (1998a), Gerber and Shiu (2006), and the references therein.

In practice, every single insurance claim is usually accompanied by a certain amount of business cost resulting from claim appraisal, investigation, settlement negotiation, and so on. The actual cost of a claim to an insurer may be different from the size of the claim.

We let $T_{i}, i=1,2, \ldots$, be the time of the $i$ th claim in the surplus process, and we denote the cost of individual claim $i$ by $\varpi\left(X_{T_{i}-}, X_{T_{i}}\right)$, which depends on the surplus just before the claim and the surplus at the claim. Thus, the expected present value of total claim costs up to the time of ruin is given by

$$
C(x)=\mathrm{E}^{x}\left[\sum_{i=1}^{N} \exp \left\{-\delta T_{i}\right\} \varpi\left(X_{T_{i}-}, X_{T_{i}}\right)\right],
$$

where $N=\max \left\{n: T_{n} \leq \tau\right\}$ is the number of claims up to ruin and $\varpi(x, y)$ is a function defined on $[0, \infty) \times(-\infty, \infty)$ representing the cost at claim.

It is easy to see that the Gerber-Shiu function $m$ is a special case of the function $C$ if ruin in the surplus process occurs only at claim times. In fact, let the function $\varpi$ in (1.2) be of the form

$$
\varpi(x, y)= \begin{cases}0 & \text { for } y \geq 0 \\ w(x,-y) & \text { for } y<0 .\end{cases}
$$

Thus, if ruin occurs or $\tau<\infty$, then $N<\infty, T_{N}=\tau, X_{T_{i}} \geq 0$ for all $i=1, \ldots, N-1$, and $X_{T_{N}}<0$. Hence,

$$
\sum_{i=1}^{N} \exp \left\{-\delta T_{i}\right\} \varpi\left(X_{T_{i}-}, X_{T_{i}}\right)=\exp \left\{-\delta T_{N}\right\} \varpi\left(X_{T_{N}-}, X_{T_{N}}\right)=\mathrm{e}^{-\delta \tau} w\left(X_{\tau-},\left|X_{\tau}\right|\right)
$$

Furthermore, if ruin does not occur or $\tau=\infty$, then $X_{T_{i}} \geq 0$ for all $i=1,2, \ldots$ and $\sum_{i=1}^{N} \exp \left\{-\delta T_{i}\right\} \varpi\left(X_{T_{i}-}, X_{T_{i}}\right)=0$. Hence,

$$
\sum_{i=1}^{N} \exp \left\{-\delta T_{i}\right\} \varpi\left(X_{T_{i}-}, X_{T_{i}}\right)=\mathrm{e}^{-\delta \tau} w\left(X_{\tau},\left|X_{\tau}\right|\right) \mathbf{1}(\tau<\infty)
$$

which means that the Gerber-Shiu function $m$ is a special form of the function $C$ with the special cost function in (1.3). Indeed, the Gerber-Shiu function only takes into account the cost or penalty at the time of ruin.

In most work in ruin theory, researchers are interested in the quantities pertaining to the time of ruin, which is the first passage time of a surplus process downcrossing 0 . However, in practice, we may also pay attention to the first passage time of a surplus process downcrossing 
any given level. In particular, such a given level is associated to the probability of default in credit risk analysis. Thus, more generally, we study the first passage time of a surplus process downcrossing a given level $d$, which is called the time of default and defined by

$$
\tau_{d}=\inf \{t: X(t)<d\}, \quad d \in(-\infty, \infty),
$$

with the convention that $\inf \{\varnothing\}=\infty$.

Clearly, $\tau_{0}=\tau$ is the time of ruin and $\tau_{-c / r}$ is the time of absolute ruin in the compound Poisson risk model with premium rate $c>0$ and borrowing rate $r>0$. References to absolute ruin can be found in Gerber (1979) and Asmussen (2000).

Besides the costs at claim times, we are also interested in the operating costs associated with a surplus process at any time throughout the life of the business. Such a cost is used in a general sense and may represent any monetary amount such as claims, penalties, dividends, utilities, and so on. We denote the operating cost at time $t$ by $l\left(X_{t}\right)$ and, thus, the expectation of the total discounted operating costs up to default is expressed as

$$
H(x)=\mathrm{E}^{x}\left[\int_{0}^{\tau_{d}} \mathrm{e}^{-\delta t} l\left(X_{t}\right) \mathrm{d} t\right],
$$

where $l$ defined on $(-\infty, \infty)$ is called the cost function.

It is easy to see that the function $H$ in (1.4) includes the expected total discounted dividends up to ruin as a special case. In fact, if $d=0$ and $l\left(X_{t}\right)$ is taken to be the dividend rate paid at time $t$, then the function $H$ in (1.4) reduces to $V$ in (1.1), the expected total discounted dividends up to ruin. For instance, with the dividend threshold $b$ and dividend rate $\alpha$, the cost function $l$ is expressed as

$$
l(x)= \begin{cases}\alpha & \text { if } x \geq b, \\ 0 & \text { if } 0 \leq x<b .\end{cases}
$$

Furthermore, if $l$ is chosen to be a utility function then the function $H$ is the expectation of the total discounted utility up to default, which has been used by Hipp and Plum (2003) as an optimization investment criterion for insurance companies.

The functions $C$ in (1.2) and $H$ in (1.4) appear to be of a different form. However, we shall prove that the function $C$ is a special case of the function $H$ in a general class of surplus processes. Therefore, it suffices to study the function $H$. As illustrated in the paper, the solution for the function $H$ will serve as a unifying tool to find solutions for many ruin-related quantities.

In this paper we will study the properties of the function $H$ and consider its applications in risk theory. As an illustration, we will focus on a piecewise-deterministic compound Poisson risk process. This risk model includes many well-known risk models in the literature such as compound Poisson risk models with dividends, interest, absolute ruin, multiple thresholds, twosided jumps, and their dual models. More general piecewise-deterministic Markov processes and their applications in ruin theory can be found in Davis (1984), (1993), Rolski et al. (1999), Dassios and Embrechts (1989), Embrechts and Schmidli (1994), Wang et al. (2003a), (2003b), and the references therein.

The rest of this paper is organized as follows. We introduce in Section 2 the piecewisedeterministic compound Poisson process. In Section 3, the relationship of the functions $C$ in (1.2) and $H$ in (1.4) is discussed and an integro-differential equation for $H$ is derived with the underlying piecewise-deterministic compound Poisson process. We give a general solution to $H$ in the classical compound Poisson process in Section 4. In the following sections, we shall investigate various applications of the total discounted operating costs up to default, namely, 
the accumulated utility up to ruin in Section 5, the total discounted claim costs up to ruin in Section 6, the probability of absolute ruin with varying borrowing rates in Section 7, and the Gerber-Shiu function with two-sided jumps, which yields the pricing formula for a perpetual American put option with two-sided jumps, is studied in Section 8. In Appendix A we provide the proofs of the results in Section 3, and the definition of the Dickson-Hipp operator and its properties used for this paper.

\section{Piecewise-deterministic compound Poisson process}

In the classical compound Poisson model, the dynamics of a surplus process $X_{t}$ is given by

$$
\mathrm{d} X_{t}=c \mathrm{~d} t-\mathrm{d} Z_{t}, \quad t \geq 0,
$$

where $c>0$ is the premium income rate and the aggregate claim $Z_{t}=\sum_{i=1}^{N_{t}} Y_{i}$ is a random sum of insurance claims defined as follows. The occurrence of insurance claims follows a Poisson process $N_{t}$ with intensity rate $\lambda$. All claim sizes $Y_{1}, Y_{2}, \ldots$ are independent and identically distributed nonnegative random variables with common distribution $Q$. Note that the surplus between any two consecutive claims satisfies the differential equation $\mathrm{d} X_{t}=c \mathrm{~d} t$.

Various modified compound Poisson risk models have appeared in the literature. Many of them are characterized by their sample paths between any two consecutive claims. We list the following few.

- If the positive surplus earns interest at rate $\rho>0$ (see Sundt and Teugels (1995)) then the surplus between any two consecutive claims satisfies the differential equation $\mathrm{d} X_{t}=$ $\left(\rho X_{t}+c\right) \mathrm{d} t$.

- If the dividend is paid at rate $0<\alpha<c$ over a threshold level $b$ (see Gerber and Shiu (2006)) then the surplus between any two consecutive claims satisfies the differential equation

$$
\mathrm{d} X_{t}= \begin{cases}(c-\alpha) \mathrm{d} t, & X_{t} \geq b, \\ c \mathrm{~d} t, & 0 \leq X_{t}<b .\end{cases}
$$

- In the dual model of the compound Poisson risk model (see Avanzi et al. (2007)), the surplus between any two consecutive claims satisfies the differential equation $\mathrm{d} X_{t}=$ $-c \mathrm{~d} t$.

All these models and many other risk models modified from the compound Poisson risk model belong to the following class of piecewise-deterministic compound Poisson processes.

Definition 2.1. A piecewise-deterministic compound Poisson (PDCP) process is a real-valued random process $\left\{X_{t}, 0 \leq t<\infty\right\}$, defined on a probability space $(\Omega, \mathcal{F}, \mathrm{P})$, satisfying the following properties.

1. $X_{0}=x$.

2. Let $T_{0}=0$ and $T_{1}, T_{2}, T_{3}, \ldots$ denote the sequence of jump points of the process $X_{t}$. Then the adapted counting process defined by $N(t)=\sum_{i=1}^{\infty} \mathbf{1}\left(T_{i} \leq t\right)$ follows a homogeneous Poisson process with intensity rate $\lambda$.

3. The jump sizes $\Delta X_{T_{k}}=X_{T_{k}}-X_{T_{k}}$ for $k=1,2,3, \ldots$ are independent and identically distributed random variables with common distribution function $Q(y)=1-\bar{Q}(y)=$ $\mathrm{P}\left\{\Delta X_{T_{1}} \leq y\right\},-\infty<y<\infty$. 
4. The process between any two consecutive jumps is deterministic and given by

$$
\left\{X_{t}=\phi_{X_{T_{k}}}(t), T_{k}<t<T_{k+1}\right\}, \quad k=0,1,2, \ldots,
$$

where $\phi_{z}(t)$ is determined by

$$
\mathrm{d} \phi_{z}(t)=g\left(\phi_{z}(t)\right) \mathrm{d} t, \quad t>0,
$$

satisfying $\phi_{z}(0)=z$ and $\lim _{t \rightarrow \infty} \phi_{z}(t)=L \in \overline{\mathbb{R}}=[-\infty, \infty]$, the function $g(x)$, $x \in B$, is locally Lipschitz continuous on each subinterval on a finite partition of its domain $B$.

Note that jumps in the PDCP process may be downward, upward, or two sided. The class of PDCP processes includes many interesting risk models appearing in the literature such as the compound Poisson risk models with interest, absolute ruin, dividend, multiple threshold, and their dual models. The corresponding expressions for $g(x)$ and $\phi_{x}(t)$ for these specific models are as follows.

- In the classical compound Poisson model, the deterministic piece between any two consecutive claims is given by $g(x)=c, x \geq 0$. Hence, $\phi_{x}(t)=x+c t, x \geq 0$, and $L=\infty$.

- In the modification of the compound Poisson model where all positive surplus earns interest at rate $\rho>0$, the corresponding function $g(x)=\rho x+c, x \geq 0$. Hence, $\phi_{x}(t)=x \mathrm{e}^{\rho t}+c \bar{s}_{\bar{t} \mid \rho}=(x+c / \rho) \mathrm{e}^{\rho t}-c / \rho, t \geq 0$, and $L=\infty$, where $\bar{s}_{\bar{t} \mid \rho}=\left(\mathrm{e}^{\rho t}-1\right) / \rho$.

- In the dividend threshold model, the corresponding function

$$
g(x)= \begin{cases}c-\alpha, & x \geq b, \\ c, & 0 \leq x<b,\end{cases}
$$

which yields

$$
\phi_{x}(t)= \begin{cases}b+(c-\alpha) t, & t \geq(b-x) / c \\ x+c t, & t<(b-x) / c\end{cases}
$$

with the limit $L=\infty$.

- In the dual model, it is characterized by $g(x)=-c, x \geq 0$, and $\phi_{x}(t)=x-c t, x \geq 0$, with $L=-\infty$.

- Furthermore, in the compound Poisson risk model where the positive surplus earns interest at rate $r>0$ and an insurer would borrow money at a borrowing rate $\rho>0$ for the negative surplus (see Zhu and Yang (2008)), the surplus follows the PDCP process with

$$
g(x)= \begin{cases}\rho x+c, & x \geq 0, \\ r x+c, & -c / r<x<0 .\end{cases}
$$

Correspondingly, we have, for $x \geq 0$,

$$
\phi_{x}(t)=x \mathrm{e}^{\rho t}+c \bar{s}_{\bar{t} \rho}=\left(x+\frac{c}{\rho}\right) \mathrm{e}^{\rho t}-\frac{c}{\rho}, \quad t \geq 0,
$$


and, for $-c / r<x<0$,

$$
\phi_{x}(t)= \begin{cases}c \bar{s}_{\overline{t-t_{0} \rho} \rho}=\frac{c}{\rho} \exp \left\{\rho\left(t-t_{0}\right)\right\}-\frac{c}{\rho}, & t \geq t_{0}, \\ x \mathrm{e}^{r t}+c \bar{s}_{\bar{t} \mid r}=\left(x+\frac{c}{r}\right) \mathrm{e}^{r t}-\frac{c}{r}, & 0 \leq t<t_{0},\end{cases}
$$

where $t_{0}=(1 / r) \log (c /(r x+c))$. In this model, we have $L=\infty$.

- In the compound Poisson risk model with multiple threshold levels $0<b_{1}<\cdots<$ $b_{n}<\infty$ and dividend rates $0<\alpha_{1}, \ldots, \alpha_{n}<c$ for corresponding thresholds respectively (see Lin and Pavlova (2006) and Lin and Sendova (2008)), it can be easily shown that the corresponding function $g(x)$ satisfies $g(x)=c-\alpha_{i}$ for $b_{i-1} \leq x<b_{i}$, $i=1, \ldots, n+1$, with $b_{0}=0, \alpha_{0}=0$, and $b_{n+1}=\infty$. The corresponding $\phi_{x}(t)=b_{i-1}+\alpha_{i} t$ for $b_{i-1} \leq \phi_{x}(t)<b_{i}$, and it is easy to verify that $L=\infty$.

- It is worthwhile pointing out that a commonly used model under the category of a piecewise-deterministic compound Poisson process with a finite limit $L$ is the modified compound Poisson model with dividend barrier $b$ where

$$
g(x)= \begin{cases}0, & x=b \\ c, & 0 \leq x<b\end{cases}
$$

Correspondingly, when $0 \leq x<b$,

$$
\phi_{x}(t)= \begin{cases}b, & t \geq(b-x) / c \\ x+c t, & 0 \leq t<(b-x) / c\end{cases}
$$

with the limit $L=b$.

\section{Relationship between the functions $C$ and $H$ and the integro-differential equation for $H$}

We shall first demonstrate that the total discounted claim costs $C$ are a special case of the total discounted operating costs $H$.

Proposition 3.1. If the surplus process $X_{t}$ is a PDCP process then the function $C$ in (1.2) can be expressed in the form of the function $H$ in (1.4) with $d=0$ and the following special cost function:

$$
l(x)=\lambda \int_{-\infty}^{\infty} \varpi(x, x+y) \mathrm{d} Q(y),
$$

namely,

$$
C(x)=\mathrm{E}^{x}\left[\int_{0}^{\tau_{0}} \mathrm{e}^{-\delta t} \lambda \int_{-\infty}^{\infty} \varpi\left(X_{t}, X_{t}+y\right) \mathrm{d} Q(y) \mathrm{d} t\right] .
$$

Proof. See Appendix A.

Since the function $C$ is a special case of the function $H$, it suffices to study the function $H$. We first derive the integro-differential equation for the function $H$, then solve the integrodifferential equation and consider its applications, which show that the function $H$ and its solution can be used as a unified approach to solving many ruin-related quantities in risk theory. 
Theorem 3.1. Suppose that the surplus process $\left\{X_{t}, t \geq 0\right\}$ is a PDCP process and that the cost function l satisfies

$$
\mathrm{E}^{x}\left[\int_{0}^{\tau_{d}} \mathrm{e}^{-\delta t}\left|l\left(X_{t}\right)\right| \mathrm{d} t\right]<\infty \text { for any } x>d .
$$

Then the function $H$ is absolutely continuous and satisfies the integro-differential equation

$$
g(x) H^{\prime}(x)-(\lambda+\delta) H(x)+\lambda \int_{d-x}^{\infty} H(x+y) \mathrm{d} Q(y)+l(x)=0, \quad x \notin D, x \in B,
$$

where $D$ is the set of all discontinuities of $l$ and $g$, and $B$ is the domain of the function $g$.

Proof. See Appendix A.

Remark 3.1. In the following frequently used cases in the literature we can simplify the expression of the integral $\int_{d-x}^{\infty} H(x+y) \mathrm{d} Q(y)$ in (3.3). Let $Q^{+}$and $Q^{-}$be distributions supported on $[0, \infty)$ representing sizes of upward jumps and downward jumps, respectively.

1. When the process $X_{t}$ has only downward jumps such that $Q(y)=1-Q^{-}(-y) \mathbf{1}(y \leq 0)$, then

$$
\int_{d-x}^{\infty} H(x+y) \mathrm{d} Q(y)=\int_{0}^{x-d} H(x-y) \mathrm{d} Q^{-}(y) .
$$

2. When the process $X_{t}$ has only upward jumps such that $Q(y)=Q^{+}(y) \mathbf{1}(y \geq 0)$, then

$$
\int_{d-x}^{\infty} H(x+y) \mathrm{d} Q(y)=\int_{0}^{\infty} H(x+y) \mathrm{d} Q^{+}(y) .
$$

3. When the process $X_{t}$ has two-sided jumps such that

$$
Q(y)=\pi Q^{+}(y) \mathbf{1}(y \geq 0)+(1-\pi)\left(1-Q^{-}(-y) \mathbf{1}(y<0)\right)
$$

with $0 \leq \pi \leq 1$, then

$$
\begin{aligned}
& \int_{d-x}^{\infty} H(x+y) \mathrm{d} Q(y) \\
& \quad=\pi \int_{0}^{\infty} H(x+y) \mathrm{d} Q^{+}(y)+(1-\pi) \int_{0}^{x-d} H(x-y) \mathrm{d} Q^{-}(y) .
\end{aligned}
$$

Since many ruin-related quantities can be expressed in the form of the function $H$ by choosing specific cost functions $l$ and by identifying the function $g$ in a PDMP risk model, we can immediately obtain integro-differential equations for these ruin-related quantities in a unified approach provided by Theorem 3.1. We shall illustrate the applications of the function $H$ together with Theorem 3.1 to consider a number of ruin-related quantities.

\section{Solution to the function $\boldsymbol{H}$ in the classical compound Poisson model}

Note that in the classical compound Poisson model, the claim size distribution $Q$ represents the downward jump distribution $Q^{-}$in Remark 3.1. For brevity, we shall slightly abuse the notation and regard $Q$ as $Q^{-}$in the classical compound Poisson model. In this case we shall denote $\tilde{q}(s)=\int_{0}^{\infty} \mathrm{e}^{-s y} \mathrm{~d} Q(y)$ and $\mu=\int_{0}^{\infty} y \mathrm{~d} Q(y)$ as the Laplace transform and the mean of the claim size distribution, respectively. 
Assume that a unique nonnegative root $\gamma$ satisfies the Lundberg equation

$$
c \gamma-(\lambda+\delta)+\lambda \tilde{q}(\gamma)=0
$$

Define

$$
\pi=\mathcal{T}_{\gamma} \bar{Q}(0)=\int_{0}^{\infty} \mathrm{e}^{-\gamma y} \bar{Q}(y) \mathrm{d} y
$$

and

$$
\bar{Q}_{\delta}(x)=\frac{1}{\pi} \mathcal{T}_{\gamma} \bar{Q}(x)=\frac{1}{\pi} \mathrm{e}^{\gamma x} \int_{x}^{\infty} \mathrm{e}^{-\gamma y} \bar{Q}(y) \mathrm{d} y,
$$

where the Dickson-Hipp operator $\mathcal{T}$ is defined in Appendix A, and $\bar{Q}(x)=1-Q(x)$ and $\bar{Q}_{\delta}(x)=1-Q_{\delta}(x)$ are the survival functions of the distribution functions $Q(x)$ and $Q_{\delta}(x)$, respectively.

Note that $\gamma \geq 0$. Thus, $0 \leq \pi \leq \mu=\int_{0}^{\infty} \bar{Q}(y) \mathrm{d} y$. Furthermore, if $c>\lambda \pi$, we define the compound geometric distribution with underlying distribution $Q_{\delta}(y)$ by

$$
G_{\delta}(x)=\sum_{k=0}^{\infty}\left(1-\frac{\lambda \pi}{c}\right)\left(\frac{\lambda \pi}{c}\right)^{k} Q_{\delta}^{* k}(x),
$$

where $Q_{\delta}^{* k}(x)$ is the $k$-fold convolution of $Q_{\delta}(x)$ with itself.

Theorem 4.1. Suppose that the surplus process $\left\{X_{t}, t \geq 0\right\}$ is the classical compound Poisson process, for which the unique root $\gamma$ of (4.1) exists and $c>\lambda \pi$, and that the cost function $l$ satisfies (3.2). The function $H$ with $d=0$ satisfies the defective renewal equation

$$
H(x)=\frac{\lambda \pi}{c} H * Q_{\delta}(x)+\frac{1}{c} \mathcal{T}_{\gamma} l(x), \quad x \geq 0,
$$

and $H$ has the solution

$$
H(x)=\frac{1}{c-\lambda \pi} \int_{0}^{x} \mathcal{T}_{\gamma} l(x-y) \mathrm{d} G_{\delta}(y), \quad x \geq 0 .
$$

Proof. In view of (3.4) and the fact that $g(x) \neq 0$ for all $x$, and noting that in this case the constant $t_{d}=\infty$ and $\phi_{x}(t)=x+c t$ in (A.3), we obtain, from (A.3),

$$
\begin{aligned}
H(x)= & \int_{0}^{\infty} \lambda \mathrm{e}^{-\lambda t} \int_{0}^{t} \mathrm{e}^{-\delta s} l(x+c s) \mathrm{d} s \mathrm{~d} t \\
& +\int_{0}^{\infty} \lambda \mathrm{e}^{-(\lambda+\delta) t} \int_{0}^{(x+c t)} H(x+c t-y) \mathrm{d} Q(y) \mathrm{d} t \\
= & \int_{0}^{\infty} \mathrm{e}^{-(\lambda+\delta) t} l(x+c t) \mathrm{d} t+\int_{0}^{\infty} \lambda \mathrm{e}^{-(\lambda+\delta) t} \int_{0}^{(x+c t)} H(x+c t-y) \mathrm{d} Q(y) \mathrm{d} t .
\end{aligned}
$$

Substituting $u=x+c t$ gives

$$
H(x)=\frac{1}{c} \int_{x}^{\infty} \mathrm{e}^{-(\lambda+\delta)(u-x) / c} l(u) \mathrm{d} u+\frac{\lambda}{c} \int_{x}^{\infty} \mathrm{e}^{-(\lambda+\delta)(u-x) / c} \int_{0}^{u} H(u-y) \mathrm{d} Q(y) \mathrm{d} u,
$$

which can be represented in terms of the Dickson-Hipp operator:

$$
H(x)=\frac{\lambda}{c} \mathcal{T}_{(\lambda+\delta) / c}\left\{H * Q+\frac{1}{c} l\right\}(x) .
$$


It follows from Lemma A.1 that

$$
\begin{aligned}
H(x) & =\frac{\lambda}{c} \mathcal{T}_{\gamma}\left\{H * Q+\frac{1}{c} l\right\}(x)-\left(\frac{\lambda+\delta}{c}-\gamma\right) \frac{\lambda}{c} \mathcal{T}_{\gamma} \mathcal{T}_{(\lambda+\delta) / c}\left\{H * Q+\frac{1}{c} l\right\}(x) \\
& =\frac{\lambda}{c} \mathcal{T}_{\gamma}\left\{H * Q+\frac{1}{c} l\right\}(x)-\left(\frac{\lambda+\delta}{c}-\gamma\right) \mathcal{T}_{\gamma} H(x) .
\end{aligned}
$$

Note that the first term can be expressed by Lemma A.3 as

$$
\frac{\lambda}{c} \mathcal{T}_{\gamma}\left\{H * Q+\frac{1}{c} l\right\}(x)=\frac{\lambda \pi}{c} H * Q_{\delta}(x)+\frac{1}{c} \mathcal{T}_{\gamma} l(x)+\frac{\lambda}{c} \tilde{q}(\gamma) \mathcal{T}_{\gamma} H(x) .
$$

Recall from (4.1) that

$$
\frac{\lambda}{c} \tilde{q}(\gamma)=\frac{\lambda+\delta}{c}-\gamma
$$

Together with (4.3) and (4.4) we obtain a defective renewal equation

$$
H(x)=\frac{\lambda \pi}{c} H * Q_{\delta}(x)+\frac{1}{c} \mathcal{T}_{\gamma} l(x) .
$$

Thus, the solution expression of $H$ follows immediately from Theorem 2.1 of Lin and Willmot (1999).

\section{The accumulated utility of an insurer}

When a risk process is used to model and assess a line of insurance business, an insurer might be interested in a quantitative measure of the overall performance in maintaining its surplus reserve. The accumulated utility up to default provides such a tool to quantify an insurer's satisfaction gained from surplus at each moment throughout the life of the business. The expectation of the accumulated utility up to the time of default is defined by

$$
U(x)=\mathrm{E}^{x}\left[\int_{0}^{\tau_{d}} u\left(X_{t}\right) \mathrm{d} t\right]
$$

where $d$ is a predetermined level of default for a particular line of business and $u(\cdot)$ is the utility function representing the insurer's attitude towards the current surplus.

As an illustration, we consider the classical compound Poisson risk model with $d=0$ and exponential utility function $u(x)=-\mathrm{e}^{-a x} / a$, where $a>0$ is a constant. In fact, the solutions in this section can be generalized to investigate a variety of other utility functions. More utility functions in the context of application in actuarial science can be found in Gerber and Pafumi (1998).

To study the expected accumulated utility up to ruin, it suffices to consider the function $W$ defined as

$$
W(x)=\mathrm{E}^{x}\left[\int_{0}^{\tau_{0}} \exp \left\{-a X_{t}\right\} \mathrm{d} t\right]
$$

Since $U(x)=-W(x) / a$, we shall now seek solutions to $W$.

When $\delta=0, \gamma=0$ is the unique nonnegative root of the Lundberg equation (4.1). Hence, $\mu=\pi$ in the assumption of Theorem 4.1, which means that the safety loading condition $c>\lambda \mu$ holds. It only remains to verify whether $l$ satisfies condition (3.2). 
Lemma 5.1. In the classical compound Poisson model with $c>\lambda \mu$, the function $W$ is bounded.

Proof. Recall that

$\mathrm{E}^{x}\left[\exp \left\{-a X_{t}\right\}\right]=\mathrm{E}^{x}\left[\exp \left\{-a\left(x+c t-\sum_{i=1}^{N(t)} Y_{i}\right)\right\}\right]=\mathrm{e}^{-a x-a c t} \mathrm{e}^{-\lambda t[1-\tilde{q}(-a)]}=\mathrm{e}^{-a x} \mathrm{e}^{f(-a) t}$,

where $f(s)=c s-\lambda+\lambda \tilde{q}(s)$ is the left-hand side of the Lundberg equation (4.1). Note that $c>\lambda \mu$. Thus, $f^{\prime}(0)>0$. Now that $f(0)=0$, it is easy to show by the continuity of $f^{\prime}(s)$ that there must exist a number $r<0$ arbitrarily close to 0 such that $f(y)<0$ and $f^{\prime}(y)>0$ when $r<y<0$. Therefore, for any $a>0$, we can always find $r>-a$ such that $f(r)<0$ and $f(-a)<f(r)$. Hence, for any $a>0$, we have

$$
\int_{0}^{\infty} \mathrm{E}^{x}\left[\exp \left\{-a X_{t}\right\}\right] \mathrm{d} t=\int_{0}^{\infty} \mathrm{e}^{-a x} \mathrm{e}^{f(-a) t} \mathrm{~d} t \leq \int_{0}^{\infty} \mathrm{e}^{-a x} \mathrm{e}^{f(r) t} \mathrm{~d} t=\frac{\mathrm{e}^{-a x}}{-f(r)}, \quad x \geq 0 .
$$

Thus, by the fact that $\exp \left\{-a X_{t}\right\}$ is nonnegative and Fubini's theorem, we obtain, for $x \geq 0$,

$$
\begin{aligned}
\mathrm{E}^{x}\left[\int_{0}^{\tau_{0}} \exp \left\{-a X_{t}\right\} \mathrm{d} t\right] & \leq \mathrm{E}^{x}\left[\int_{0}^{\infty} \exp \left\{-a X_{t}\right\} \mathrm{d} t\right] \\
& =\int_{0}^{\infty} \mathrm{E}^{x}\left[\exp \left\{-a X_{t}\right\}\right] \mathrm{d} t \\
& \leq \frac{\mathrm{e}^{-a x}}{-f(r)} \\
& <\frac{1}{-f(r)} .
\end{aligned}
$$

Therefore, $W(x)$ is bounded for all $x \geq 0$.

Corollary 5.1. In the classical compound Poisson model with $c>\lambda \mu$, the solution to $W$ is given by

$$
W(x)=\frac{\mathrm{e}^{-a x}}{a(c-\lambda \mu)} \int_{0}^{x} \mathrm{e}^{a y} \mathrm{~d} G_{0}(y), \quad x \geq 0,
$$

where $G_{0}$ is the compound geometric distribution defined by

$$
G_{0}(x)=\sum_{n=0}^{\infty}\left(1-\frac{\lambda \mu}{c}\right)\left(\frac{\lambda \mu}{c}\right)^{n} Q_{0}^{* n}(x)
$$

and $Q_{0}$ is defined by $Q_{0}(x)=(1 / \mu) \int_{0}^{x} \bar{Q}(y) \mathrm{d} y, x \geq 0$.

Proof. Since $W$ is bounded, then condition (3.2) is satisfied. Since $\delta=0$, we have $\gamma=0$. Applying Theorem 4.1, we obtain

$$
W(x)=\frac{1}{c-\lambda \mu} \int_{0}^{x} \mathcal{T}_{0} l(x-y) \mathrm{d} G_{0}(y), \quad x \geq 0 .
$$

Since $W(x)$ is a special case of $H(x)$ with the cost function $l(x)=\mathrm{e}^{-a x}$, we have

$$
\mathcal{T}_{0} l(y)=\int_{y}^{\infty} \mathrm{e}^{-a x} \mathrm{~d} x=\frac{1}{a} \mathrm{e}^{-a y},
$$

which leads to the desired solution, (5.1), upon substitution. 
When the claim size distribution is exponentially distributed, we can derive an explicit solution for the expected accumulated utility up to ruin, which is presented in the following result.

Corollary 5.2. In the classical compound Poisson model with $c>\lambda \mu$, if the claim size distribution $Q$ is exponential with mean $1 / \beta$ then $W$ admits an explicit solution given by

$$
W(x)=\frac{\lambda}{a c^{2}(a-\beta+\lambda / c)} \mathrm{e}^{-(\beta-\lambda / c) x}+\frac{a-\beta}{a c(a-\beta+\lambda / c)} \mathrm{e}^{-a x}, \quad x \geq 0 .
$$

Proof. If the claim size distribution $Q$ is exponential with mean $1 / \beta$ then the equilibrium distribution $Q_{0}$ is exponential with the same mean. It is well known that $G_{0}$ is an exponential function given by

$$
G_{0}(y)=1-\left(\frac{\lambda}{\beta c}\right) \mathrm{e}^{-(\beta-\lambda / c) y}, \quad y \geq 0 .
$$

Note that $G_{0}$ has a probability mass point at 0 with $G_{0}(0)=1-\lambda /(\beta c)$. Substituting (5.3) into (5.1) yields (5.2).

\section{Total discounted claim costs up to ruin}

As shown in Section 3, the total discounted claim costs up to ruin is a special case of the total discounted operating costs up to default. We are now able to derive explicit solutions for the function $C$, the total discounted claim costs up to ruin, in the classical compound Poisson model by using the solution for the function $H$.

Corollary 6.1. Under the assumptions of Theorem 4.1, the solution to $C$ is given by

$$
C(x)=\frac{1}{c-\lambda \pi} \int_{0}^{x} \zeta(x-y) \mathrm{d} G_{\delta}(y), \quad x \geq 0
$$

where

$$
\zeta(z)=\lambda \mathrm{e}^{\gamma z} \int_{z}^{\infty} \mathrm{e}^{-\gamma x} \int_{0}^{\infty} \varpi(x, x-y) \mathrm{d} Q(y) \mathrm{d} x .
$$

Proof. As a result of Theorem 4.1 and Theorem 3.1, the solution follows immediately upon substitution of $l(x)=\lambda \int_{0}^{\infty} \varpi(x, x-y) \mathrm{d} Q(y) \mathrm{d} x$.

The expected total discounted claim $\operatorname{cost} C(x)$ includes many interesting ruin-related quantities. In particular, it includes the expected discounted aggregate claims up to ruin and the expected number of claims up to ruin as special cases, which are discussed in the following two examples.

Example 6.1. (Expected discounted aggregate claims up to ruin.) To investigate the present value of future obligations for claim payments, an insurer would be interested in the discounted aggregate claims up to ruin, defined by

$$
K(x)=\mathrm{E}^{x}\left[\sum_{i=1}^{N} \exp \left\{-\delta T_{i}\right\} Y_{i}\right], \quad x \geq 0,
$$

where $\delta>0$ and $N=\max \left\{n: T_{n} \leq \tau_{0}\right\}$. It is obviously a special case of $C$ with the cost at claim $\varpi(x, y)=x-y$ and, hence, $\varpi(x, x-y)=y$. 
By Corollary 6.1, we easily obtain an explicit expression for the expected total discounted claims up to ruin with exponential claims.

Corollary 6.2. In the classical compound Poisson model, for which the nonnegative root $\gamma$ exists for the Lundberg equation and $c>\lambda \pi$, the solution to $K$ is given by

$$
K(x)=\frac{\lambda \mu}{(c-\lambda \pi) \gamma} G_{\delta}(x), \quad x \geq 0 .
$$

Proof. In this case, it follows from (3.1) that

$$
l(x)=\lambda \int_{0}^{\infty} y \mathrm{~d} Q(y)=\lambda \mu,
$$

which satisfies (3.2) since $K$ is bounded by $\lambda \mu / \delta$. Furthermore, the expression for $\zeta(x)$ in (6.1) reduces to $\zeta(x)=\lambda \mu / \gamma$ and, hence, the desired result is obtained.

Corollary 6.3. If $Q(y)$ is exponentially distributed with mean $1 / \beta$, the expected total discounted claims up to ruin $K$ is given by

$$
K(x)=\frac{\lambda}{\delta \beta}\left(1-\frac{\rho+\beta}{\beta} \mathrm{e}^{\rho x}\right), \quad x \geq 0,
$$

where

$$
\rho=\frac{(\lambda+\delta-\beta c)-\sqrt{(\lambda+\delta-\beta c)^{2}+4 \delta \beta c}}{2 c} .
$$

Proof. In the case where $Q(y)=1-\mathrm{e}^{-\beta y}$, we can easily show that $G_{\delta}$ defined in (4.2) reduces to

$$
G_{\delta}(x)=1-\frac{\lambda}{(\beta+\gamma) c} \exp \left\{-\left(\beta-\frac{\lambda \beta}{(\beta+\gamma) c}\right) y\right\}
$$

where $\gamma$ is the positive root to the Lundberg equation (4.1), which reduces to

$$
c s^{2}+(\beta c-\lambda-\delta) s-\delta \beta=0 .
$$

We shall denote the negative root of (6.4) by $\rho$. Hence, it follows by substitution of $G_{\delta}$ in (6.2) that

$$
K(x)=\frac{\lambda(\beta+\gamma)}{(c(\beta+\gamma)-\lambda) \beta \gamma}\left(1-\frac{\lambda}{(\beta+\gamma) c} \exp \left\{-\left(\beta-\frac{\lambda \beta}{(\beta+\gamma) c}\right) y\right\}\right), \quad x \geq 0 .
$$

Since $\gamma$ is a root of (6.4), then

$$
\delta=\frac{(c(\beta+\gamma)-\lambda) \gamma}{\beta+\gamma} .
$$

Note that $\rho \gamma=\delta \beta / c$. Hence,

$$
\rho=\frac{\delta \beta}{c \gamma}=\frac{(c(\beta+\gamma)-\lambda) \beta}{c(\beta+\gamma)}=\frac{\lambda \beta}{(\beta+\gamma) c}-\beta .
$$

Therefore, we obtain (6.3) by substitution of parameters. 
We point out that the moments of the total discounted claims until a given time in a renewal risk process are studied in Léveillé and Garrido (2001a), (2001b). However, their results do not apply to the expectation of the total discounted claims up to ruin.

Example 6.2. (Expected number of claims up to ruin.) Another special case of the expected total discounted claim costs is the expected number of claims up to ruin defined by

$$
N(x)=\mathrm{E}^{x}[N],
$$

which is a special case of the function $C$ in (1.2), letting $\delta=0$ and $\varpi(x, y)=1$.

It follows immediately from (3.1) that $l(x)=\lambda$. By Proposition 3.1 we see that

$$
N(x)=\mathrm{E}^{x}\left[\lambda \tau_{0}\right]=\lambda \mathrm{E}^{x}\left[\tau_{0}\right] .
$$

Note that if $c>\lambda \mu$ then $\mathrm{P}^{x}\left\{\tau_{0}<\infty\right\}<1$ or, equivalently, $\mathrm{P}^{x}\left\{\tau_{0}=\infty\right\}>0$. Thus, $\mathrm{E}^{x}\left[\tau_{0}\right]=\infty$, which in turn leads to $N(x)=\infty$. Therefore, in order to find an explicit solution to the function $N$, we consider the $c<\lambda \mu$ case, under which condition (3.2) can be verified.

Lemma 6.1. In the classical compound Poisson model for which $c<\lambda \mu$, the function $N(x)$, the expected number of claims up to ruin, satisfies, for $x \geq 0$,

$$
N(x) \leq M \mathrm{e}^{a x} \text { for all } a>0 \text { and some constant } M,
$$

and, hence, condition (3.2) is satisfied.

Proof. By definition we have, for any $a>0$,

$$
\mathrm{E}^{x}\left[\exp \left\{a X_{t}\right\}\right]=\exp \left\{a\left(x+c t-\sum_{i=1}^{N(t)} Y_{i}\right)\right\}=\mathrm{e}^{a x} \mathrm{e}^{f(a) t}
$$

where $f(s)=c s-\lambda+\lambda \tilde{q}(s)$.

Note that $f(0)=0$ and $f^{\prime}(0)<0$ since $c<\lambda \mu$. Thus, we can find an arbitrarily small $a>0$ such that $f(a)<0$. Thus, $\lambda \leq \lambda \mathrm{e}^{a x}$ for any such $a>0$. Therefore,

$$
\begin{aligned}
\mathrm{E}^{x}\left[\int_{0}^{\tau_{0}}\left|l\left(X_{t}\right)\right| \mathrm{d} t\right] & =\mathrm{E}^{x}\left[\int_{0}^{\tau_{0}} \lambda \mathrm{d} t\right] \\
& \leq \mathrm{E}^{x}\left[\int_{0}^{\tau_{0}} \lambda \exp \left\{a X_{t}\right\} \mathrm{d} t\right] \\
& =\lambda \mathrm{E}^{x}\left[\int_{0}^{\infty} \exp \left\{a X_{t}\right\} \mathrm{d} t\right] \\
& =\lambda \int_{0}^{\infty} \mathrm{E}^{x}\left[\exp \left\{a X_{t}\right\}\right] \mathrm{d} t \\
& =\lambda \mathrm{e}^{a x} \int_{0}^{\infty} \mathrm{e}^{f(a) t} \mathrm{~d} t \\
& =M \mathrm{e}^{a x} \\
& <\infty,
\end{aligned}
$$

where $M=-\lambda / f(a)$.

Note that $\mathrm{e}^{a x}$ is increasing in $a$ for $x \geq 0$. Hence, (6.6) holds for all $a>0$ and, thus, the desired result is obtained. 
Not only does Lemma 6.1 verify condition (3.2), which enables us to apply Theorem 3.1, but it also leads to the result that

$$
\mathrm{e}^{-a x} N(x)<\text { constant for all } x \geq 0 .
$$

Corollary 6.4. In the classical compound Poisson model, if the safety loading factor $\theta=$ $(\beta c-\lambda) / \lambda<0$ then the expected number of claims up to ruin $N(x)$ in (6.5) is given by

$$
N(x)=\frac{\lambda \beta}{\lambda-\beta c} x+\frac{\lambda}{\lambda-\beta c} .
$$

Proof. Applying Theorem 3.1, we obtain, by letting $\varpi(x, y)=1$ and $\delta=0$ in (3.3),

$$
c N^{\prime}(x)-\lambda N(x)+\lambda \beta \int_{0}^{x} N(x-y) \mathrm{e}^{-\beta y} \mathrm{~d} y+\lambda=0, \quad x \geq 0 .
$$

By taking derivatives on both sides of (6.8), we obtain

$$
c N^{\prime \prime}(x)+(\beta c-\lambda) N^{\prime}(x)+\lambda \beta=0 .
$$

By inspection we see that $N(x)=A x$ is a particular solution, where $A$ is a constant satisfying $(\beta c-\lambda) A+\lambda \beta=0$, which shows that $A=\lambda \beta /(\lambda-\beta c)$. Thus, the general solution to (6.9) is given by

$$
N(x)=\frac{\lambda \beta}{\lambda-\beta c} x+A_{1}+A_{2} \mathrm{e}^{(\lambda / c-\beta) x}, \quad x \geq 0,
$$

since $s=0$ and $s=\lambda / c-\beta$ are the two roots of the characteristic equation $c s^{2}+(\beta c-\lambda) s=0$. Inserting the expression for $N(x)$ in (6.10) into (6.8) gives

$$
\begin{aligned}
& \frac{\lambda \beta c}{\lambda-\beta c}+A_{2}(\lambda-\beta c) \mathrm{e}^{(\lambda / c-\beta) x}-\lambda\left(\frac{\lambda \beta}{\lambda-\beta c} x+A_{1}+A_{2} \mathrm{e}^{(\lambda / c-\beta) x}\right) \\
& +\lambda \beta \int_{0}^{x}\left(\frac{\lambda \beta}{\lambda-\beta c} y+A_{1}+A_{2} \mathrm{e}^{(\lambda / c-\beta) y}\right) \mathrm{e}^{-\beta(x-y)} \mathrm{d} y+\lambda \\
& \quad=0
\end{aligned}
$$

which implies that

$$
\begin{aligned}
& \frac{\lambda \beta c}{\lambda-\beta c}+A_{2}(\lambda-\beta c) \mathrm{e}^{(\lambda / c-\beta) x}-\frac{\lambda^{2} \beta}{\lambda-\beta c} x-\lambda A_{1}-\lambda A_{2} \mathrm{e}^{(\lambda / c-\beta) x}+\frac{\lambda^{2} \beta}{\lambda-\beta c} x-\frac{\lambda^{2}}{\lambda-\beta c} \\
& +\frac{\lambda^{2}}{\lambda-\beta c} \mathrm{e}^{-\beta x}+\lambda A_{1}-\lambda A_{1} \mathrm{e}^{-\beta x}+c \beta A_{2} \mathrm{e}^{(\lambda / c-\beta) x}-c \beta A_{2} \mathrm{e}^{-\beta x}+\lambda \\
& \quad=0 .
\end{aligned}
$$

Upon rearrangement and cancellation, the above equation is simplified to

$$
\frac{\lambda^{2}}{\lambda-\beta c} \mathrm{e}^{-\beta x}-\lambda A_{1} \mathrm{e}^{-\beta x}-c \beta A_{2} \mathrm{e}^{-\beta x}=0 .
$$

Hence, the undetermined coefficients $A_{1}$ and $A_{2}$ must satisfy

$$
\frac{\lambda^{2}}{\lambda-\beta c}-\lambda A_{1}-c \beta A_{2}=0 \text {. }
$$


Recall from (6.7) that there exists a number $a \in(0, \lambda / c-\beta)$ such that

$$
\mathrm{e}^{-a x} N(x)=\frac{\lambda \beta}{\lambda-\beta c} x \mathrm{e}^{-a x}+A_{1} \mathrm{e}^{-a x}+A_{2} \mathrm{e}^{(\lambda / c-\beta-a) x}<\text { constant for all } x \geq 0 .
$$

Hence, we must have $A_{2}=0$. It follows from (6.11) that

$$
A_{1}=\frac{\lambda}{\lambda-\beta c}
$$

Therefore, the desired result is obtained.

\section{Absolute ruin with varying borrowing rates}

In a modification of the classical compound Poisson risk model, we may allow a certain line of insurance business to continue even when the surplus falls below 0 . When it does fall below 0 , an insurer borrows loans from external sources at a prescribed borrowing rate.

In the classical absolute ruin models, it is often assumed that an insurer loans at a constant borrowing rate $r$ as soon as the surplus becomes negative, $X(t)<0$. In this case, the absolute ruin occurs when the negative surplus reaches the level $-c / r$. In other words, if $X(t)<-c / r$ or $r X(t)+c<0$, the premium collected at rate $c$ is no longer able to even cover the interest due at rate $-r X(t)$. Absolute ruin problems with constant borrowing rate have been well studied in the literature. See, for example, Asmussen (2000), Cai (2007), Gerber and Yang (2007), Wang and Yin (2009), Yang et al. (2008), Yuan and Hu (2008), Zhang and Wu (1999), Zhu and Yang (2008), and the references therein.

In a more general case, we assume that the borrowing rate $r(y), y<0$, is a piecewise locally Lipschitz continuous function in the negative surplus level $y$. It is important to note that absolute ruin occurs at the new level $d$, which is the largest root of the equation

$$
r(y) y+c=0, \quad y<0 .
$$

We call $d$ the level of absolute ruin because the premium income is no longer able to cover the borrowing interest when $X_{t}<d$. Therefore, the surplus between any two consecutive claims satisfies

$$
\mathrm{d} X_{t}= \begin{cases}c \mathrm{~d} t, & \text { if } X_{t} \geq 0, \\ \left(r\left(X_{t}\right) X_{t}+c\right) \mathrm{d} t, & \text { if } d<X_{t}<0,\end{cases}
$$

where $d<0$ is the largest root of (7.1). Hence, as an example of the PDCP process, we can express the growth of surplus by

$$
g(x)= \begin{cases}c & \text { if } x \geq 0, \\ r(x) x+c & \text { if } d<x<0 .\end{cases}
$$

Accordingly, the time of absolute ruin is defined by $\tau_{d}=\inf \left\{t: X_{t}<d\right\}$. The expected discounted penalty at absolute ruin or the Gerber-Shiu function at absolute ruin is defined by

$$
m_{\mathrm{abs}}(x)=\mathrm{E}^{x}\left[\exp \left\{-\delta \tau_{d}\right\} w\left(X_{\tau_{d}-},\left|X_{\tau_{d}}\right|\right) \mathbf{1}\left(\tau_{d}<\infty\right)\right],
$$

where $w(x, y)$ is a nonnegative function defined on $[d, \infty) \times(|d|, \infty)$ representing the penalty at absolute ruin. If condition (3.2) is satisfied, it follows from Theorem 3.1 that the Gerber-Shiu 
function $m_{\text {abs }}$ satisfies

$$
\begin{aligned}
& c m_{\mathrm{abs}}^{\prime}(x)-(\lambda+\delta) m_{\mathrm{abs}}(x)+\lambda \int_{0}^{x-d} m_{\mathrm{abs}}(x-y) \mathrm{d} Q(y)+\lambda \int_{x-d}^{\infty} w(x, y-x) \mathrm{d} Q(y) \\
& \quad=0, \quad x \geq 0,
\end{aligned}
$$

and

$$
\begin{aligned}
& (r(x) x+c) m_{\mathrm{abs}}^{\prime}(x)-(\lambda+\delta) m_{\mathrm{abs}}(x)+\lambda \int_{0}^{x-d} m_{\mathrm{abs}}(x-y) \mathrm{d} Q(y) \\
& +\lambda \int_{x-d}^{\infty} w(x, y-x) \mathrm{d} Q(y) \\
& \quad=0, \quad d<x<0 .
\end{aligned}
$$

To simplify our analysis, we investigate a special case of the Gerber-Shiu function $m_{\mathrm{abs}}$ where $\delta=0$, denoted by

$$
\varphi_{\mathrm{abs}}(x)=\mathrm{E}^{x}\left[w\left(X_{\tau_{d}-},\left|X_{\tau_{d}}\right|\right) \mathbf{1}\left(\tau_{d}<\infty\right)\right],
$$

where $w$ is assumed to be bounded, implying that (3.2) is satisfied.

The special function $\varphi_{\text {abs }}$ still includes many interesting quantities such as the absolute ruin probability, the distribution functions of the surplus before absolute ruin, the surplus at absolute ruin, the surplus causing absolute ruin, and many more.

If the claim size distribution $Q$ follows an exponential distribution, we can solve the integrodifferential equations (7.2) and (7.3) for an explicit solution as follows.

Corollary 7.1. If $Q$ is exponentially distributed with mean $1 / \beta, \varphi_{\mathrm{abs}}$ admits an explicit solution given by

$$
\begin{aligned}
& \varphi_{\mathrm{abs}}(x)=C_{1}+\int_{0}^{x} \mathrm{e}^{-S(y)}\left(C_{2}+\int_{0}^{y} \mathrm{e}^{S(t)} f(t) \mathrm{d} t\right) \mathrm{d} y, \quad x \geq 0, \\
& \varphi_{\mathrm{abs}}(x)=C_{3}+\int_{d}^{x} \mathrm{e}^{-G(y)}\left(C_{4}+\int_{d}^{y} \mathrm{e}^{G(t)} h(t) \mathrm{d} t\right) \mathrm{d} y, \quad d<x<0,
\end{aligned}
$$

where

$$
\begin{aligned}
& S(y)=\int_{0}^{y}\left(\beta-\frac{\lambda}{c}\right) \mathrm{d} t=\left(\beta-\frac{\lambda}{c}\right) y, \\
& f(x)=-\frac{\beta \zeta(x)+\zeta^{\prime}(x)}{c} \\
& \zeta(x)=\lambda \beta \int_{x-d}^{\infty} \mathrm{e}^{-\beta y} w(x, y-x) \mathrm{d} y \\
& G(y)=\int_{d}^{x} g(t) \mathrm{d} t \\
& g(x)=\frac{r^{\prime}(x) x+r(x)+\beta r(x) x+\beta c-\lambda}{r(x) x+c} \\
& h(x)=-\frac{\beta \zeta(x)+\zeta^{\prime}(x)}{r(x) x+c}
\end{aligned}
$$


and the coefficients are determined by

$$
\begin{aligned}
& C_{1}=-\int_{0}^{\infty} \mathrm{e}^{-S(y)}\left(C_{2}+\int_{0}^{y} \mathrm{e}^{S(t)} f(t) \mathrm{d} t\right) \mathrm{d} y, \\
& C_{2}= \mathrm{e}^{-G(0)} C_{4}+\mathrm{e}^{-G(0)} \int_{d}^{0} \mathrm{e}^{G(t)} h(t) \mathrm{d} t, \\
& C_{3}=\zeta(d), \\
& C_{4}=-\left(\zeta(d)+\int_{0}^{\infty} \mathrm{e}^{-S(y)} \int_{0}^{y} \mathrm{e}^{S(t)} f(t) \mathrm{d} t \mathrm{~d} y+\int_{d}^{0} \mathrm{e}^{-G(y)} \int_{d}^{y} \mathrm{e}^{G(t)} h(t) \mathrm{d} t \mathrm{~d} y\right. \\
&\left.\quad+\mathrm{e}^{-G(0)} \int_{0}^{d} \mathrm{e}^{-G(t)} h(t) \mathrm{d} t \int_{0}^{\infty} \mathrm{e}^{-S(y)} \mathrm{d} y\right) \\
& \quad \times\left(\int_{d}^{0} \mathrm{e}^{-G(y)} \mathrm{d} y+\mathrm{e}^{-G(0)} \int_{0}^{\infty} \mathrm{e}^{-S(y)} \mathrm{d} y\right)^{-1} .
\end{aligned}
$$

Proof. Differentiating both sides of (7.2) and (7.3), and making substitutions yields

$$
\begin{aligned}
& \varphi_{\mathrm{abs}}^{\prime \prime}(x)+\left(\beta-\frac{\lambda}{c}\right) \varphi_{\mathrm{abs}}^{\prime}(x)=f(x), \quad x \geq 0, x \notin D, \\
& \varphi_{\mathrm{abs}}^{\prime \prime}(x)+g(x) \varphi_{\mathrm{abs}}^{\prime}(x)=h(x), \quad d<x<0, x \notin D,
\end{aligned}
$$

where $D$ is the set of points of $x$, at which $r(x)$ is not differentiable.

The general solution to $\varphi_{\mathrm{abs}}(x)$ is given by (7.4) and (7.5). In order to determine those coefficients, we search for four boundary conditions, each of which gives a linear equation involving the coefficients.

Since $c>\lambda \mu$ and $w$ is bounded, we have

$$
\lim _{x \rightarrow \infty} \varphi_{\mathrm{abs}}(x)=0 .
$$

Letting $x \rightarrow d$ in (7.3) yields

$$
\varphi_{\mathrm{abs}}(d+)=\zeta(d)
$$

By the continuity of $\varphi_{\mathrm{abs}}$ at 0 we have

$$
\varphi_{\mathrm{abs}}(0-)=\varphi_{\mathrm{abs}}(0+) .
$$

Letting $x=0$ in (7.2) and $x \rightarrow 0$ in (7.3), and in view of (7.8) we obtain

$$
\varphi_{\mathrm{abs}}^{\prime}(0-)=\varphi_{\mathrm{abs}}^{\prime}(0+) \text {. }
$$

Hence, inserting (7.4) and (7.5) into (7.6), (7.7), (7.8), and (7.9) yields the desired solutions.

\section{The Gerber-Shiu function with two-sided jumps}

In the classical compound Poisson model jumps are downward only whereas in its dual model jumps are upward only. However, it is also interesting to consider the compound Poisson model with two-sided jumps. For instance, Gerber and Shiu (1996) used such a two-sided jump model in asset pricing. With two-sided jumps, we assume that random events arrive according to the Poisson process $N_{t}$. For each event, it brings either a random insurance claim with common 
distribution $Q^{-}$or a random investment income (cash injection) with common distribution $Q^{+}$. The probability of the event being an insurance claim is assumed to be $\pi$ and, thus, the event happens to be an investment income with the chance $1-\pi$. Therefore, the jump size distribution is given by (3.5).

In view of (3.3) and (3.6), the integro-differential equation for $H$ in the compound Poisson risk model with two-sided jumps would be

$$
\begin{aligned}
& c H^{\prime}(x)-(\lambda+\delta) H(x)+\lambda \pi \int_{0}^{\infty} H(x+y) \mathrm{d} Q^{+}(y) \\
& +\lambda(1-\pi) \int_{0}^{x} H(x-y) \mathrm{d} Q^{-}(y)+l(x) \\
& \quad=0 .
\end{aligned}
$$

It is difficult to solve the integro-differential equation (8.1). However, we can derive explicit solutions to (8.1) for the double exponential jumps, where the distribution function of the jump size is given by

$$
Q(y)=\pi\left(1-\exp \left\{-\beta_{1} y\right\}\right) \mathbf{1}(y \geq 0)+(1-\pi)\left[1-\left(1-\exp \left\{-\beta_{2} y\right\}\right) \mathbf{1}(y<0)\right],
$$

where $\beta_{1}>0, \beta_{2}>0$, and $0 \leq \pi \leq 1$.

Readers are referred to Kou and Wang (2003) for related topics in a more general jump diffusion process with two-sided exponential jumps.

With the double exponential distribution (8.2), the integro-differential equation (8.1) becomes

$$
\begin{aligned}
& c H^{\prime}(x)-(\lambda+\delta) H(x)+\lambda \pi \beta_{1} \exp \left\{\beta_{1} x\right\} \int_{x}^{\infty} H(y) \exp \left\{-\beta_{1} y\right\} \mathrm{d} y \\
& +\lambda(1-\pi) \beta_{2} \exp \left\{-\beta_{2} x\right\} \int_{0}^{x} H(y) \exp \left\{\beta_{2} y\right\} \mathrm{d} y+l(x) \\
& \quad=0 .
\end{aligned}
$$

As an illustration, we solve the following special case of the Gerber-Shiu function, denoted by $\psi_{\delta}$ :

$$
\psi_{\delta}(x)=\mathrm{E}^{x}\left[\exp \left\{-\delta \tau_{0}\right\} f\left(\left|X_{\tau_{0}}\right|\right) \mathbf{1}\left(\tau_{0}<\infty\right)\right],
$$

where $f$ is a bounded function defined on $(0, \infty)$.

The special function $\psi_{\delta}$ includes the absolute ruin probability, the Laplace transform of the absolute ruin time, the distribution function of the surplus at absolute ruin, the moments of the surplus at absolute ruin, and so on.

Corollary 8.1. For $\delta>0$, if $Q$ is the double exponential distribution (8.2), $\psi_{\delta}$ admits an explicit solution given by

$$
\psi_{\delta}(x)=\left(\left(\beta_{2}+\rho\right) \int_{0}^{\infty} f(z) \exp \left\{-\beta_{2} z\right\} \mathrm{d} z\right) \mathrm{e}^{-\rho x}, \quad x \geq 0,
$$

where $\rho$ is the unique negative root of the Lundberg fundamental equation

$$
c s+\lambda\left(\pi \frac{\beta_{1}}{\beta_{1}-s}+(1-\pi) \frac{\beta_{2}}{\beta_{2}+s}-1\right)=\delta .
$$


Proof. Taking derivatives with respect to $x$ and making a substitution in (8.3) gives

$$
\begin{aligned}
& c H^{\prime \prime}(x)-(\lambda+\delta) H^{\prime}(x)-c \beta_{1} H^{\prime}(x)+\beta_{1}(\lambda+\delta) H(x) \\
& -\beta_{1} \lambda(1-\pi) \beta_{2} \exp \left\{-\beta_{2} x\right\} \int_{0}^{x} H(y) \exp \left\{\beta_{2} y\right\} \mathrm{d} y-\beta_{1} l(x)-\lambda \pi \beta_{1} H(x) \\
& +c \beta_{2} H^{\prime}(x)-\beta_{2}(\lambda+\delta) H(x)+\beta_{2} \lambda \pi \beta_{1} \exp \left\{\beta_{1} x\right\} \int_{x}^{\infty} H(y) \exp \left\{-\beta_{1} y\right\} \mathrm{d} y \\
& +\beta_{2} l(x)+\lambda(1-\pi) \beta_{2} H(x)+l^{\prime}(x) \\
& \quad=0 .
\end{aligned}
$$

Taking derivatives with respect to $x$ again and substituting the integral terms yields

$$
\begin{aligned}
& c H^{\prime \prime \prime}(x)+\left(c \beta_{2}-c \beta_{1}-\lambda-\delta\right) H^{\prime \prime}(x) \\
& +\left[\lambda(1-\pi) \beta_{2}-\lambda \pi \beta_{1}-\beta_{2}(\lambda+\delta)+\beta_{1}(\lambda+\delta)-c \beta_{1} \beta_{2}\right] H^{\prime}(x) \\
& +\delta \beta_{1} \beta_{2} H(x)+l^{\prime \prime}(x)+\left(\beta_{2}-\beta_{1}\right) l^{\prime}(x)-\beta_{1} \beta_{2} l(x) \\
& \quad=0 .
\end{aligned}
$$

Note that $w(x, y-x)=f(y-x)$. Then

$$
\begin{aligned}
l(x) & =\lambda(1-\pi) \beta_{2} \int_{x}^{\infty} f(y-x) \exp \left\{-\beta_{2} y\right\} \mathrm{d} y \\
& =\lambda(1-\pi) \beta_{2} \exp \left\{-\beta_{2} x\right\} \int_{0}^{\infty} f(z) \exp \left\{-\beta_{2} z\right\} \mathrm{d} z .
\end{aligned}
$$

It is easy to verify that in this case $l^{\prime \prime}(x)+\left(\beta_{2}-\beta_{1}\right) l^{\prime}(x)-\beta_{1} \beta_{2} l(x)=0$. Thus, (8.1) and (8.5) reduce to

$$
\begin{aligned}
& c \psi_{\delta}^{\prime}(x)-(\lambda+\delta) \psi_{\delta}(x)+\lambda \pi \int_{0}^{\infty} \psi_{\delta}(x+y) \mathrm{d} Q^{+}(y)+\lambda(1-\pi) \int_{0}^{x} \psi_{\delta}(x-y) \mathrm{d} Q^{-}(y) \\
& +\lambda(1-\pi) \beta_{2} \exp \left\{-\beta_{2} x\right\} \int_{0}^{\infty} f(z) \exp \left\{-\beta_{2} z\right\} \mathrm{d} z \\
& \quad=0
\end{aligned}
$$

and

$$
\begin{aligned}
& c \psi_{\delta}^{\prime \prime \prime}(x)+\left(c \beta_{2}-c \beta_{1}-\lambda-\delta\right) \psi_{\delta}^{\prime \prime}(x) \\
& +\left[\lambda(1-\pi) \beta_{2}-\lambda \pi \beta_{1}-\beta_{2}(\lambda+\delta)+\beta_{1}(\lambda+\delta)-c \beta_{1} \beta_{2}\right] \psi_{\delta}^{\prime}(x)+\delta \beta_{1} \beta_{2} \psi_{\delta}(x) \\
& \quad=0 .
\end{aligned}
$$

We know that the fundamental solution to $\psi_{\delta}$ can be written as

$$
C_{1} \mathrm{e}^{\rho x}+C_{2} \exp \left\{\gamma_{1} x\right\}+C_{3} \exp \left\{\gamma_{2} x\right\}
$$

where $\rho \leq 0, \gamma_{1} \geq 0$, and $\gamma_{2}>\gamma_{1}$ are the three real roots of the characteristic function

$$
\begin{aligned}
& c s^{3}+\left(c \beta_{2}-c \beta_{1}-\lambda-\delta\right) s^{2} \\
& +\left[\lambda(1-\pi) \beta_{2}-\lambda \pi \beta_{1}-\beta_{2}(\lambda+\delta)+\beta_{1}(\lambda+\delta)-c \beta_{1} \beta_{2}\right] s+\delta \beta_{1} \beta_{2} \\
& \quad=0,
\end{aligned}
$$

which is equivalent to the Lundberg fundamental equation (8.4). 
We denote the left-hand side of (8.4) by $k(s)$. Note that $\delta>0$. It is obvious from (8.4) that $k(0)=0$ and $k\left(-\beta_{2}+\right)=+\infty$; hence, there must be one solution $\rho \in\left(-\beta_{2}, 0\right)$ for $k(s)=\delta$. We also have a solution $\gamma_{1} \in\left(0, \beta_{1}\right)$ as $k\left(\beta_{1}-\right)=+\infty$, and a solution $\gamma_{2} \in\left(\beta_{1},+\infty\right)$ as $k\left(\beta_{1}+\right)=-\infty$ and $k(+\infty)=+\infty$.

Since $\lim _{u \rightarrow+\infty} \psi_{\delta}(u)=0$, we must have $C_{2}=C_{3}=0$, i.e.

$$
\psi_{\delta}(x)=C_{1} \mathrm{e}^{\rho x} .
$$

Substituting this into (8.6) gives

$$
\begin{aligned}
& c \rho C_{1} \mathrm{e}^{\rho x}-(\lambda+\delta) C_{1} \mathrm{e}^{\rho x}+\lambda \pi \beta_{1} \exp \left\{\beta_{1} x\right\} \int_{x}^{\infty} C_{1} \exp \left\{-\left(\beta_{1}-\rho\right) y\right\} \mathrm{d} y \\
& +\lambda \pi \beta_{2} \exp \left\{-\beta_{2} x\right\} \int_{0}^{x} C_{1} \exp \left\{\left(\beta_{2}+\rho\right) y\right\} \mathrm{d} y+\lambda(1-\pi) \exp \left\{-\beta_{2} x\right\} \\
& \quad=0 .
\end{aligned}
$$

Rearranging terms yields,

$$
\begin{aligned}
& {\left[c \rho-(\lambda+\delta)+\frac{\lambda \pi \beta_{1}}{\beta_{1}-\rho}+\frac{\lambda(1-\pi) \beta_{2}}{\beta_{2}+\rho}\right] C_{1} \mathrm{e}^{\rho x}-\frac{\lambda(1-\pi) \beta_{2} C_{1}}{\beta_{2}+\rho} \exp \left\{-\beta_{2} x\right\}} \\
& +\left(\lambda(1-\pi) \beta_{2} \int_{0}^{\infty} f(z) \exp \left\{-\beta_{2} z\right\} \mathrm{d} z\right) \exp \left\{-\beta_{2} x\right\} \\
& \quad=0 .
\end{aligned}
$$

Note that the algebraic expression in square brackets is Lundberg's equation and, hence, vanishes. Therefore,

$$
C_{1}=\left(\beta_{2}+\rho\right) \int_{0}^{\infty} f(z) \exp \left\{-\beta_{2} z\right\} \mathrm{d} z .
$$

When $\delta=0, \psi_{\delta}$ reduces to the case

$$
\psi(x)=\mathrm{E}^{x}\left[f\left(\left|X_{\tau_{0}}\right|\right) \mathbf{1}\left(\tau_{0}<\infty\right)\right] .
$$

Corollary 8.2. If $Q$ is the double exponential distribution (8.2), $\psi$ admits an explicit solution given by

$$
\psi(x)= \begin{cases}\left(\left(\beta_{2}+\rho\right) \int_{0}^{\infty} f(z) \exp \left\{-\beta_{2} z\right\} \mathrm{d} z\right) \mathrm{e}^{\rho x} & \text { if } \theta>0, \\ \frac{\int_{0}^{\infty} f(z) \exp \left\{-\beta_{2} z\right\} \mathrm{d} z}{\beta_{2}} & \text { if } \theta \leq 0,\end{cases}
$$

where the safety loading factor $\theta=(c-\lambda \mu) /(\lambda \mu), \mu=\lambda\left[(1-\pi) / \beta_{2}-\pi / \beta_{1}\right]$, and

$$
\rho=\frac{\left(\lambda-c \beta_{2}+c \beta_{1}\right)-\Delta}{2 c}
$$

with

$$
\Delta=\sqrt{\left(\lambda-c \beta_{2}+c \beta_{1}\right)^{2}-4 c\left[\lambda(1-\pi) \beta_{2}-\lambda \pi \beta_{1}-\beta_{2} \lambda+\beta_{1} \lambda-c \beta_{1} \beta_{2}\right]} .
$$

Proof. It is not hard to see that the Lundberg equation reduces to

$$
c s^{3}+\left(c \beta_{2}-c \beta_{1}-\lambda-\delta\right) s^{2}+\left[\lambda(1-\pi) \beta_{2}-\lambda \pi \beta_{1}-\beta_{2} \lambda+\beta_{1} \lambda-c \beta_{1} \beta_{2}\right] s=0 .
$$


It is obvious that $s=0$ is a solution and the other two roots are given by

$$
\gamma=\frac{\left(\lambda-c \beta_{2}+c \beta_{1}\right)+\Delta}{2 c} \text { and } \rho=\frac{\left(\lambda-c \beta_{2}+c \beta_{1}\right)-\Delta}{2 c} .
$$

We can show that if $c>\lambda \mu=\lambda\left[(1-\pi) / \beta_{2}-\pi / \beta_{1}\right]$ then $\rho<0$ and $\gamma>0$. Otherwise, $\gamma>\rho>0$. If the negative root $\rho$ to the Lundberg equation exists, the result follows from Corollary 8.1.

When the Lundberg equation does not have a negative solution, we must have $\psi(x)=C_{1}$, where $C_{1}$ is determined from the integro-differential equation

$$
\begin{aligned}
& c \psi^{\prime}(x)-\lambda \psi(x)+\lambda \pi \int_{0}^{\infty} \psi(x+y) \mathrm{d} Q^{+}(y)+\lambda(1-\pi) \int_{0}^{x} \psi(x-y) \mathrm{d} Q^{-}(y) \\
& +\left(\lambda(1-\pi) \beta_{2} \int_{0}^{\infty} f(z) \exp \left\{-\beta_{2} z\right\} \mathrm{d} z\right) \exp \left\{-\beta_{2} x\right\} \\
& \quad=0 .
\end{aligned}
$$

We find out that in this case

$$
C_{1}=\frac{1}{\beta_{2}} \int_{0}^{\infty} f(z) \exp \left\{-\beta_{2} z\right\} \mathrm{d} z
$$

Since

$$
l^{\prime}(0)=c+\lambda \pi \frac{1}{\beta_{1}}-\lambda(1-\pi) \frac{1}{\beta_{2}}
$$

and recall that

$$
c=(1+\theta) \mathrm{E}[Y]=(1+\theta)\left(\lambda \pi \frac{1}{\beta_{1}}-\lambda(1-\pi) \frac{1}{\beta_{2}}\right),
$$

whether (8.7) has a negative or zero solution depends solely on $\theta$.

Note that when $\theta \leq 0$, the safety loading condition is violated and the surplus process has a negative drift; hence, ruin is deemed to occur ultimately. If we take $f(y)=1$ in $\psi$ then $\psi(x)=\mathrm{E}^{x}\left[\mathbf{1}\left(\tau_{0}<\infty\right)\right]=1$.

\subsection{A perpetual American put option with two-sided jumps}

In Gerber and Shiu (1996), (1998b), it was successfully demonstrated that the Gerber-Shiu discounted penalty function can be applied to price a perpetual American put option. The underlying stock price is driven by an exponential of a shifted compound Poisson process. It turns out that the price of a perpetual American option is the discounted expected value of a payoff function, which can be regarded as a penalty function, evaluated at an optimal hitting time. In the same line of logic, we shall derive the price of a perpetual American put option based on a underlying stock price driven by an exponential of a compound Poisson process with two-sided jumps. To obtain explicit solutions, we need as given the assumption of two-sided exponential jumps.

Assume that the stock price process is given by

$$
S(t)=\mathrm{e}^{X(t)}=\exp \left\{x+c t+\sum_{i=1}^{N(t)} Y_{i}\right\}
$$

where $c$ is the expected yield rate, the counting process $\{N(t), t \geq 0\}$ is Poisson process with intensity $\lambda$, and the sequence of random movements $\left\{Y_{i}, i=1,2, \ldots\right\}$ are mutually independent 
and follow the common distribution

$$
Q(y)=\pi\left(1-\exp \left\{-\beta_{1} y\right\}\right) \mathbf{1}(y \geq 0)+(1-\pi)\left[1-\left(1-\exp \left\{-\beta_{2} y\right\}\right) \mathbf{1}(y<0)\right] .
$$

It is known in mathematical finance that the price of an American put option is given by

$$
P(x)=\sup _{a} \mathrm{E}^{x}\left[\exp \left\{-\delta \tau_{a}\right\} \Pi\left(S\left(\tau_{a}\right)\right)\right]=\sup _{a} \mathrm{E}^{x}\left[\exp \left\{-\delta \tau_{a}\right\} \Pi\left(\exp \left\{X\left(\tau_{a}\right)\right\}\right)\right],
$$

where the payoff function

$$
\Pi(s)=(K-s)_{+},
$$

$K$ is the exercise price, and

$$
\tau_{a}=\inf \left\{t: S(t)<\mathrm{e}^{a}\right\}=\inf \{t: X(t)<a\}
$$

with $a<\ln K \leq x$. Now we are able to derive an analogous result with the perpetual American put option with only negative jumps obtained in Gerber and Shiu (1998).

Corollary 8.3. When $\delta>0$, the solution to $F(x)$ in (8.8) is given by

$$
P(x)=\frac{\left(\beta_{2}+\rho\right) K}{\beta_{2}(1-\rho)}\left(K \frac{\rho\left(\beta_{2}+1\right)}{\beta_{2}(\rho-1)}\right)^{-\rho} \mathrm{e}^{\rho x},
$$

where $\rho$ is the unique negative solution to (8.4).

When $\delta=0$, the solution to $F(x)$ is given by

$$
P(x)= \begin{cases}\frac{\left(\beta_{2}+\rho\right) K}{\beta_{2}(1-\rho)}\left(K \frac{\rho\left(\beta_{2}+1\right)}{\beta_{2}(\rho-1)}\right)^{-\rho} \mathrm{e}^{\rho x} & \text { if } \theta>0, \\ \frac{K}{\beta_{2}^{2}} & \text { if } \theta \leq 0 .\end{cases}
$$

Proof. If we define a new process $Y=\left\{Y_{t}, t \geq 0\right\}$ such that $Y_{t}=X_{t}-a$ and its corresponding time of default $\tau_{d}^{Y}=\inf \{t: Y(t)<d\}$, then it is easy to see that $\tau_{0}^{Y}=\tau_{a}$. We have to keep in mind that $Y(0)=x-a$. Therefore, the discounted payoff function upon which the supremum is taken can be written as a special case of $\psi_{\delta}(x)$ :

$$
\mathrm{E}^{x}\left[\exp \left\{-\delta \tau_{a}\right\} \Pi\left(\exp \left\{X\left(\tau_{a}\right)\right\}\right)\right]=\mathrm{E}^{x-a}\left[\exp \left\{-\delta \tau_{0}^{Y}\right\} \Pi\left(\exp \left\{Y\left(\tau_{0}^{Y}\right)+a\right\}\right)\right] .
$$

When $\delta>0$, it follows immediately from Corollary 8.1 that

$$
\mathrm{E}^{x}\left[\exp \left\{-\delta \tau_{a}\right\} \Pi\left(\exp \left\{X\left(\tau_{a}\right)\right\}\right)\right]=\left(K-\frac{\beta_{2}}{\beta_{2}+1} \mathrm{e}^{a}\right) \frac{\beta_{2}+\rho}{\beta_{2}} \mathrm{e}^{\rho(x-a)},
$$

which is maximized at

$$
a=\ln \left(K \frac{\rho\left(\beta_{2}+1\right)}{\beta_{2}(\rho-1)}\right) .
$$

Since $\rho \in\left(-\beta_{2}, 0\right)$, we can show that $a<\ln K$.

When $\delta=0$, a similar result follows from Corollary 8.2. When $\theta \leq 0$,

$$
\mathrm{E}^{x}\left[\exp \left\{-\delta \tau_{a}\right\} \Pi\left(\exp \left\{X\left(\tau_{a}\right)\right\}\right)\right]=\frac{K}{\beta_{2}^{2}}-\frac{\mathrm{e}^{a}}{\beta_{2}\left(\beta_{2}-1\right)},
$$

which is maximized at $a=-\infty$. This completes the proof.

The last part of the corollary makes sense because the investor is better off delaying exercising the option as late as possible, as the safety loading condition $\theta>0$ is violated and the stock price process will eventually drift towards 0 . 


\section{Appendix A}

Proof of Proposition 3.1. First, we define a counting process associated with $X_{t}$ by

$$
N(t, A)=\sum_{i=1}^{\infty} \mathbf{1}\left(T_{i} \leq t\right) \mathbf{1}\left(X_{T_{i}}-X_{T_{i}-} \in A\right), \quad t \geq 0,
$$

where $A$ is a subset of $(-\infty, \infty)$. Obviously, $N(t, A)$ is a Poisson process from the definition of the PDCP process. Hence, its compensator process denoted by $\tilde{N}(t, A)$ is given by $\tilde{N}(t, A)=$ $\lambda Q(A) t, t \geq 0$.

Then, in terms of the associated counting process measure $N(\mathrm{~d} t, \mathrm{~d} y)$, the expectation in (1.2) can also be written as

$$
C(x)=\mathrm{E}^{x}\left[\int_{0}^{\tau_{0}} \int_{-\infty}^{\infty} \mathrm{e}^{-\delta t} \varpi\left(X_{t-}, X_{t-}+y\right) N(\mathrm{~d} t, \mathrm{~d} y)\right] .
$$

By similar arguments used in Section 2 of Chapter II of Brémaud (1981), we know that the expectation in (A.1) can be expressed as the expectation in terms of the compensator process measure, namely,

$$
\begin{aligned}
C(x) & =\mathrm{E}^{x}\left[\int_{0}^{\tau_{0}} \int_{-\infty}^{\infty} \mathrm{e}^{-\delta t} \varpi\left(X_{t-}, X_{t-}+y\right) N(\mathrm{~d} t, \mathrm{~d} y)\right] \\
& =\mathrm{E}^{x}\left[\int_{0}^{\tau_{0}} \int_{-\infty}^{\infty} \mathrm{e}^{-\delta t} \varpi\left(X_{t-}, X_{t-}+y\right) \tilde{N}(\mathrm{~d} t, \mathrm{~d} y)\right] \\
& =\mathrm{E}^{x}\left[\int_{0}^{\tau_{0}} \mathrm{e}^{-\delta t} \lambda \int_{-\infty}^{\infty} \varpi\left(X_{t-}, X_{t-}+y\right) \mathrm{d} Q(y) \mathrm{d} t\right] \\
& =\mathrm{E}^{x}\left[\int_{0}^{\tau_{0}} \mathrm{e}^{-\delta t} \lambda \int_{-\infty}^{\infty} \varpi\left(X_{t}, X_{t}+y\right) \mathrm{d} Q(y) \mathrm{d} t\right] .
\end{aligned}
$$

The last equality can be explained as follows. Since the process $X_{t}$ can only have countable discontinuities, then, for each $\omega \in \Omega,\left\{t: X_{t-}(\omega) \neq X_{t}(\omega)\right\}$ is a countable set. Hence, almost surely,

$$
\begin{aligned}
\int_{0}^{\tau_{0}} & \mathrm{e}^{-\delta t} \lambda \int_{-\infty}^{\infty} \varpi\left(X_{t}(\omega), X_{t}(\omega)+y\right) \mathrm{d} Q(y) \mathrm{d} t \\
& =\int_{0}^{\tau_{0}} \mathrm{e}^{-\delta t} \lambda \int_{-\infty}^{\infty} \varpi\left(X_{t-}(\omega), X_{t-}(\omega)+y\right) \mathrm{d} Q(y) \mathrm{d} t .
\end{aligned}
$$

Proof of Theorem 3.1. Let $T=T_{1} \wedge t_{d}$, where $t_{d}=\inf \left\{t \mid \phi_{x}(t)<d\right\}$, with the convention that $\inf \{\varnothing\}=\infty$. Note that $T$ is a stopping time with respect to $\left\{\mathcal{F}_{t}\right\}$. Then,

$$
H(x)=\mathrm{E}^{x}\left[\int_{0}^{T} \mathrm{e}^{-\delta s} l\left(X_{s}\right) \mathrm{d} s\right]+\mathrm{E}^{x}\left[\int_{T}^{\tau_{d}} \mathrm{e}^{-\delta s} l\left(X_{s}\right) \mathrm{d} s\right] .
$$

Note that when $T_{1}>t_{d}$, we have $\tau_{d}=t_{d}=T$. While $T_{1} \leq t_{d}$, we have $T=T_{1}$. Thus,

$$
\begin{aligned}
\mathrm{E}^{x}\left[\int_{T}^{\tau_{d}} \mathrm{e}^{-\delta s} l\left(X_{s}\right) \mathrm{d} s\right]= & \mathrm{E}^{x}\left[\mathbf{1}\left(T_{1}>t_{d}\right) \int_{T}^{\tau_{d}} \mathrm{e}^{-\delta s} l\left(X_{s}\right) \mathrm{d} s\right] \\
& +\mathrm{E}^{x}\left[\mathbf{1}\left(T_{1} \leq t_{d}\right) \int_{T}^{\tau_{d}} \mathrm{e}^{-\delta s} l\left(X_{s}\right) \mathrm{d} s\right] \\
= & \mathrm{E}^{x}\left[\mathbf{1}\left(T_{1} \leq t_{d}\right) \int_{T_{1}}^{\tau_{d}} \mathrm{e}^{-\delta s} l\left(X_{s}\right) \mathrm{d} s\right]
\end{aligned}
$$


where

$$
\mathrm{E}^{x}\left[\mathbf{1}\left(T_{1} \leq t_{d}\right) \int_{T_{1}}^{\tau_{d}} \mathrm{e}^{-\delta s} l\left(X_{s}\right) \mathrm{d} s\right]=\mathrm{E}^{x}\left[\mathrm{E}^{x}\left[\int_{T_{1}}^{\tau_{d}} \mathbf{1}\left(T_{1} \leq t_{d}\right) \mathrm{e}^{-\delta s} l\left(X_{s}\right) \mathrm{d} s \mid \mathcal{F}_{T_{1}}\right]\right] .
$$

For any given constant $M>0$, it follows from the strong Markov property that

$$
\begin{aligned}
\mathrm{E}^{x}\left[\mathrm{E}^{x}\left[\left\{\mathbf{1}\left(T_{1} \leq t_{d}\right) \int_{T_{1}}^{\tau_{d}} \mathrm{e}^{-\delta s} l\left(X_{s}\right) \mathrm{d} s\right\} \wedge M \mid \mathcal{F}_{T_{1}}\right]\right] \\
=\mathrm{E}^{x}\left[\mathrm{E}^{X_{T_{1}}}\left[\left\{\mathbf{1}\left(T_{1} \leq t_{d}\right) \int_{0}^{\tau_{d}-T_{1}} \mathrm{e}^{-\delta\left(T_{1}+s\right)} l\left(X_{T_{1}+s}\right) \mathrm{d} s\right\} \wedge M\right]\right] .
\end{aligned}
$$

Let $Y(s)=X\left(s+T_{1}\right), s \geq 0$, and define $\tau_{d}^{Y}=\inf \{t \mid Y(t)<d, t \geq 0\}$. Note that $\tau_{d}^{Y}=\tau_{d}-T_{1}$ when $T_{1} \leq t_{d}$. Thus,

$$
\begin{array}{r}
\mathrm{E}^{x}\left[\mathrm{E}^{X_{T_{1}}}\left[\left\{\mathbf{1}\left(T_{1} \leq t_{d}\right) \int_{0}^{\tau_{d}-T_{1}} \exp \left\{-\delta\left(T_{1}+s\right)\right\} l\left(X_{T_{1}+s}\right) \mathrm{d} s\right\} \wedge M\right]\right] \\
=\mathrm{E}^{x}\left[\mathrm{E}^{X_{T_{1}}}\left[\left\{\mathbf{1}\left(T_{1} \leq t_{d}\right) \exp \left\{-\delta T_{1}\right\} \int_{0}^{\tau_{d}^{Y}} \mathrm{e}^{-\delta s} l\left(Y_{s}\right) \mathrm{d} s\right\} \wedge M\right]\right] .
\end{array}
$$

In view of (3.2), we obtain, by the dominated convergence theorem,

$$
\begin{aligned}
\mathrm{E}^{x}\left[\mathbf{1}\left(T_{1} \leq t_{d}\right) \int_{T_{1}}^{\tau_{d}} \mathrm{e}^{-\delta s} l\left(X_{s}\right) \mathrm{d} s\right] \\
\quad=\lim _{M \rightarrow \infty} \mathrm{E}^{x}\left[\mathrm{E}^{x}\left[\left\{\mathbf{1}\left(T_{1} \leq t_{d}\right) \int_{T_{1}}^{\tau_{d}} \mathrm{e}^{-\delta s} l\left(X_{s}\right) \mathrm{d} s\right\} \wedge M \mid \mathcal{F}_{T_{1}}\right]\right] \\
\quad=\lim _{M \rightarrow \infty} \mathrm{E}^{x}\left[\mathrm{E}^{X_{T_{1}}}\left[\left\{\mathbf{1}\left(T_{1} \leq t_{d}\right) \exp \left\{-\delta T_{1}\right\} \int_{0}^{\tau_{d}-T_{1}} \mathrm{e}^{-\delta s} l\left(X_{s}\right) \mathrm{d} s\right\} \wedge M\right]\right] \\
\quad=\mathrm{E}^{x}\left[\mathrm{E}^{X_{T_{1}}}\left[\mathbf{1}\left(T_{1} \leq t_{d}\right) \exp \left\{-\delta T_{1}\right\} \int_{0}^{\tau_{d}^{Y}} \mathrm{e}^{-\delta s} l\left(Y_{s}\right) \mathrm{d} s\right]\right] \\
\quad=\mathrm{E}^{x}\left[\mathbf{1}\left(T_{1} \leq t_{d}\right) \exp \left\{-\delta T_{1}\right\} H\left(X_{T_{1}}\right)\right] .
\end{aligned}
$$

Once again by the dominated convergence theorem we have

$$
\begin{aligned}
\mathrm{E}^{x}[\mathbf{1} & \left.\left(T_{1} \leq t_{d}\right) \exp \left\{-\delta T_{1}\right\} H\left(X_{T_{1}}\right)\right] \\
& =\lim _{M \rightarrow \infty} \mathrm{E}^{x}\left[\mathbf{1}\left(T_{1} \leq t_{d}\right) \exp \left\{-\delta T_{1}\right\} H\left(X_{T_{1}}\right) \wedge M\right] \\
& =\lim _{M \rightarrow \infty} \mathrm{E}^{x}\left[\mathrm{E}^{x}\left[\mathbf{1}\left(T_{1} \leq t_{d}\right) \exp \left\{-\delta T_{1}\right\} H\left(X_{T_{1}}\right) \wedge M \mid X_{T_{1}-}\right]\right] \\
& =\lim _{M \rightarrow \infty} \mathrm{E}^{x}\left[\left\{\mathbf{1}\left(T_{1} \leq t_{d}\right) \exp \left\{-\delta T_{1}\right\} \int_{-\infty}^{\infty} H\left(X_{T_{1}-}+y\right) \mathrm{d} Q(y)\right\} \wedge M\right] \\
& =\mathrm{E}^{x}\left[\mathbf{1}\left(T_{1} \leq t_{d}\right) \exp \left\{-\delta T_{1}\right\} \int_{d-X_{T_{1}-}}^{\infty} H\left(X_{T_{1}-}+y\right) \mathrm{d} Q(y)\right]
\end{aligned}
$$

where the last equality follows from the fact that $H(x)=0$ when $x<d$. 
Recall from property 4 of Definition 2.1 that $X_{T_{1}}$ is solely determined by the continuous piece $\left\{\phi_{x}(t), 0 \leq t<T_{1}\right\}$. Returning to (A.2), we now have

$$
\begin{aligned}
H(x)= & \mathrm{E}^{x}\left[\int_{0}^{T} \mathrm{e}^{-\delta s} l\left(\phi_{x}(s)\right) \mathrm{d} s\right] \\
& +\mathrm{E}^{x}\left[\mathbf{1}\left(T_{1} \leq t_{d}\right) \exp \left\{-\delta T_{1}\right\} \int_{d-\phi_{x}\left(T_{1}-\right)}^{\infty} H\left(\phi_{x}\left(T_{1}-\right)+y\right) \mathrm{d} Q(y)\right] .
\end{aligned}
$$

Recall from property 2 of Definition 2.1 that $T_{1}$ is exponentially distributed with mean $1 / \lambda$. Hence,

$$
\begin{aligned}
H(x)= & \int_{0}^{t_{d}} \lambda \mathrm{e}^{-\lambda t} \int_{0}^{t} \mathrm{e}^{-\delta s} l\left(\phi_{x}(s)\right) \mathrm{d} s \mathrm{~d} t+\int_{0}^{t_{d}} \lambda \mathrm{e}^{-(\lambda+\delta) t} \int_{d-\phi_{x}(t)}^{\infty} H\left(\phi_{x}(t)+y\right) \mathrm{d} Q(y) \mathrm{d} t \\
& +\exp \left\{-\lambda t_{d}\right\} \int_{0}^{t_{d}} \mathrm{e}^{-\delta s} l\left(\phi_{x}(s)\right) \mathrm{d} s .
\end{aligned}
$$

We now discuss the two cases in which $g(x)=0$ and $g(x) \neq 0$.

In the case where $g(x)=0$, it is easy to see that $\phi_{x}(t)=x, t \geq 0$. Hence, $t_{d}=\inf \{\varnothing\}=\infty$ since $d<x$. Thus, (A.3) reduces to

$$
\begin{aligned}
H(x) & =\int_{0}^{\infty} \lambda \mathrm{e}^{-\lambda t} \int_{0}^{t} \mathrm{e}^{-\delta s} l(x) \mathrm{d} s \mathrm{~d} t+\int_{0}^{\infty} \lambda \mathrm{e}^{-(\lambda+\delta) t} \int_{d-x}^{\infty} H(x+y) \mathrm{d} Q(y) \mathrm{d} t \\
& =\frac{1}{\lambda+\delta} l(x)+\frac{\lambda}{\lambda+\delta} \int_{d-x}^{\infty} H(x+y) \mathrm{d} Q(y),
\end{aligned}
$$

which is a special case of (3.3) when $g(x)=0$.

In the case where $g(x) \neq 0$, recall from property 3 of Definition 2.1 that $\phi_{x}(t)$ is uniquely determined by

$$
\mathrm{d} \phi_{x}(t)=g\left(\phi_{x}(t)\right) \mathrm{d} t, \quad 0<t<t_{d}
$$

with $\phi_{x}(0)=x$. Furthermore, $\phi_{x}\left(t_{d}\right)=L$ if $t_{d}=\infty$ and $\phi_{x}\left(t_{d}\right)=d$ if $t_{d}<\infty$, which holds since $\phi_{x}(t)$ is continuous in $t$. However, since neither $L$ or $d$ depends on $x$, we can denote $\phi_{x}\left(t_{d}\right)=m$, which is a constant. Thus, if we let $z=\phi_{x}(t)$ then $\mathrm{d} t=\mathrm{d} z / g(z)$ and $t_{d}=\int_{x}^{m} \mathrm{~d} z / g(z)$, and, thus, (A.3) becomes

$$
\begin{aligned}
H(x)= & \int_{x}^{m} \lambda \exp \left\{-\lambda \int_{x}^{z} \frac{1}{g(y)} \mathrm{d} y\right\} \int_{x}^{z} \exp \left\{-\delta \int_{x}^{u} \frac{1}{g(y)} \mathrm{d} y\right\} \frac{l(u)}{g(u)} \mathrm{d} u \frac{1}{g(z)} \mathrm{d} z \\
& +\int_{x}^{m} \lambda \exp \left\{-(\lambda+\delta) \int_{x}^{z} \frac{1}{g(y)} \mathrm{d} y\right\} \int_{d-z}^{\infty} H(z+y) \mathrm{d} Q(y) \frac{1}{g(z)} \mathrm{d} z \\
& +\exp \left\{-\lambda \int_{x}^{m} \frac{1}{g(y)} \mathrm{d} y\right\} \int_{x}^{m} \exp \left\{-\delta \int_{x}^{z} \frac{1}{g(y)} \mathrm{d} y\right\} \frac{l(z)}{g(z)} \mathrm{d} z
\end{aligned}
$$

which implies that $H$ is absolutely continuous. 
We denote the three terms on the right-hand side of (A.4) by $I_{1}, I_{2}$, and $I_{3}$, respectively. Thus, by taking derivatives on the both sides of (A.4), we have, for $x \notin D$,

$$
\begin{aligned}
H^{\prime}(x)= & \frac{\lambda+\delta}{g(x)} I_{1}-\frac{l(x)}{g(x)} \int_{x}^{m} \lambda \exp \left\{-\lambda \int_{x}^{z} \frac{1}{g(y)} \mathrm{d} y\right\} \frac{1}{g(z)} \mathrm{d} z+\frac{\lambda+\delta}{g(x)} I_{2} \\
& -\frac{\lambda}{g(x)} \int_{d-x}^{\infty} H(x+y) \mathrm{d} Q(y)+\frac{\lambda+\delta}{g(x)} I_{3}-\frac{l(x)}{g(x)} \exp \left\{-\lambda \int_{x}^{m} \frac{1}{g(y)} \mathrm{d} y\right\} \\
= & \frac{\lambda+\delta}{g(x)}\left(I_{1}+I_{2}+I_{3}\right)-\frac{l(x)}{g(x)}-\frac{\lambda}{g(x)} \int_{d-x}^{\infty} H(x+y) \mathrm{d} Q(y) \\
= & \frac{\lambda+\delta}{g(x)} H(x)-\frac{l(x)}{g(x)}-\frac{\lambda}{g(x)} \int_{d-x}^{\infty} H(x+y) \mathrm{d} Q(y),
\end{aligned}
$$

which implies (3.3).

Definition A.1. For any integrable function $f$ defined on $[0, \infty)$ and a real number $s \geq 0$, the Dickson-Hipp transform of $f$ is given by

$$
\mathcal{T}_{s} f(x)=\mathrm{e}^{s x} \int_{x}^{\infty} \mathrm{e}^{-s y} f(y) \mathrm{d} y, \quad x \geq 0 .
$$

The transform $\mathcal{T}_{s}$ is called the Dickson-Hipp operator, which was introduced in Dickson and Hipp (2001).

Note that the Laplace transform of $f$ is indeed a special case of the Dickson-Hipp operator

$$
\mathcal{T}_{s} f(0)=\int_{0}^{\infty} \mathrm{e}^{-s y} f(y) \mathrm{d} y .
$$

Definition A.2. For any integrable functions $f$ and $Q$ defined on $[0, \infty)$, the convolution of $f$ and $Q$ is given by

$$
f * Q(x)=\int_{0}^{x} f(x-y) \mathrm{d} Q(y), \quad x \geq 0 .
$$

For convenience, however, in the derivation of the following lemmas we shall introduce another definition of convolution. Readers may directly refer to Lemma A.3 for the major conclusion of the appendix.

Definition A.3. For any integrable functions $f$ and $q$ defined on $[0, \infty)$, the convolution of $f$ and $g$ is given by

$$
f \star q(x)=\int_{0}^{x} f(x-y) q(y) \mathrm{d} y, \quad x \geq 0 .
$$

The following properties of the Dickson-Hipp operator are used in this paper. The proofs and references to the following two lemmas can be found in Li and Garrido (2004) and Gerber and Shiu (2006).

Lemma A.1. If $s_{1} \geq 0, s_{2} \geq 0$, and $s_{1} \neq s_{2}$, then

$$
\mathcal{T}_{s_{1}} \mathcal{T}_{s_{2}} f(x)=\frac{\mathcal{T}_{s_{2}} f(x)-\mathcal{T}_{s_{1}} f(x)}{s_{1}-s_{2}} .
$$

The lemma shows that the Dickson-Hipp operators are commutable with each other. Hence, they are also commutable with Laplace transforms. 
Lemma A.2. If $s \geq 0$ then

$$
\mathcal{T}_{s}\{f \star q\}(x)=\mathcal{T}_{s} q(0) \mathcal{T}_{s} f(x)+f \star \mathcal{T}_{s} q(x) .
$$

Lemma A.3. If $Q$ is a distribution function and $s \geq 0$, then

$$
\mathcal{T}_{s}\{f * Q\}(x)=\tilde{q}(s) \mathcal{T}_{s} f(x)+f * B(x),
$$

where $\tilde{q}(s)=\int_{0}^{\infty} \mathrm{e}^{-s y} \mathrm{~d} Q(y)$ and $\bar{B}(x)=\mathcal{T}_{s} \bar{Q}(x)$.

Proof. For simplicity, we assume that $q=Q^{\prime}$. Hence, $f \star q=f * Q$ and the results follow by Lemma A.2 and the fact that $\mathcal{T}_{0} \mathcal{T}_{s} q(x)=\mathcal{T}_{s} \mathcal{T}_{0} q(x)=\mathcal{T}_{s} \bar{Q}(x)$. The proof can be generalized to a more general distribution $Q$.

\section{Acknowledgements}

The authors would like to thank the anonymous referee for helpful comments and suggestions that improved the presentation of this paper. This research was partly supported by the Natural Sciences and Engineering Research Council of Canada (NSERC), the Society of Actuaries/Casualty Actuarial Society, and the Institute of Quantitative Finance and Insurance (IQFI) at the University of Waterloo. The authors also thank the participants at the Second International Workshop on Gerber-Shiu Functions, held at the Radon Institute of the Austrian Academy of Sciences in Linz, Austria, August 27-29, 2008, for their comments on the original version of this paper.

\section{References}

Asmussen, S. (2000). Ruin Probabilities (Adv. Ser. Statist. Sci. Appl. Prob. 2). World Scientific, River Edge, NJ.

Avanzi, B., Gerber, H. U. AND ShIU, E. S. W. (2007). Optimal dividends in the dual model. Insurance Math. Econom. 41, 111-123.

Brémaud, P. (1981). Point Processes and Queues. Springer, New York.

CAI, J. (2007). On the time value of absolute ruin with debit interest. Adv. Appl. Prob. 39, 343-359.

Dassios, A. ANd Embrechts, P. (1989). Martingales and insurance risk. Commun. Statist. Stoch. Models 5, 181-217.

DAvis, M. H. A. (1984). Piecewise-deterministic Markov processes: a general class of nondiffusion stochastic models. J. R. Statist. Soc. B 46, 353-388.

Davis, M. H. A. (1993). Markov Models and Optimization (Monogr. Statist. Appl. Prob. 49). Chapman and Hall, London.

Dickson, D. C. M. AND HipP, C. (2001). On the time to ruin for Erlang(2) risk processes. Insurance Math. Econom. 29, 333-344.

Embrechts, P. ANd Schmidli, H. (1994). Ruin estimation for a general insurance risk model. Adv. Appl. Prob. 26, 404-422.

Gerber, H. U. (1979). An Introduction to Mathematical Risk Theory (S. S. Heubner Found. Monogr. Ser. 8). University of Pennsylvania, Philadelphia.

Gerber, H. U. and Pafumi, G. (1998). Utility functions: from risk theory to finance. N. Amer. Actuarial J. 2, 74-90.

Gerber, H. U. AND ShiU, E. S. W. (1996). Actuarial bridges to dynamics hedging and option pricing. Insurance Math. Econom. 18, 183-218.

Gerber, H. U. ANd Shiu, E. S. W. (1998a). On the time value of ruin. N. Amer. Actuarial J. 2, 48-78.

Gerber, H. U. and Shiu, E. S. W. (1998b). Pricing perpetual options for jump processes. N. Amer. Actuarial J. 2, 101-112.

Gerber, H. U. And ShiU, E. S. W. (2006). On optimal dividend strategies in the compound Poisson model. N. Amer. Actuarial J. 10, 76-93.

Gerber, H. U. AND YANG, H. (2007). Absolute ruin probabilities in a jump diffusion risk model with investment. N. Amer. Actuarial J. 11, 159-169.

Hipp, C. ANd Plum, M. (2003). Optimal investment for investors with state dependent income, and for insurers. Finance Stoch. 7, 299-321.

Kou, S. G. And Wang, H. (2003). First passage times of a jump diffusion process. Adv. Appl. Prob. 35, 504-531. 
LÉveillé, G. AND GARRIDO, J. (2001a). Moments of compound renewal sums with discounted claims. Insurance Math. Econom. 28, 217-231.

LÉveILlÉ, G. AND GARRIDO, J. (2001b). Recursive moments of compound renewal sums with discounted claims. Scand. Actuarial J. 2001, 98-110.

Li, S. AND GARRIDO, J. (2004). On a class of renewal risk models with a constant dividend barrier. Insurance Math. Econom. 35, 691-701.

Lin, X. S. And Pavlova, K. P. (2006). The compound Poisson risk model with a threshold dividend strategy. Insurance Math. Econom. 38, 57-80.

Lin, X. S. And Sendova, K. P. (2008). The compound Poisson model with multiple thresholds. Insurance Math. Econom. 42, 617-627.

Lin, X. S. AND Willmot, G. E. (1999). Analysis of a defective renewal equation arising in ruin theory. Insurance Math. Econom. 25, 63-84.

Rolski, T., Schmidli, H., Schmidt, V. And Teugels, J. (1999). Stochastic Processes for Insurance and Finance. John Wiley, Chichester.

Sundt, B. AND Teugels, J. L. (1995). Ruin estimates under interest force. Insurance Math. Econom. 16, 7-22.

WANG, C. AND YIN, C. (2009). Dividend payments in the classical risk model under absolute ruin with debit interest. Appl. Stoch. Models Business Industry 25, 247-262.

WANG, G.-J., QIAN, S.-P AND WU, R. (2003a). Distribution of deficit at ruin for a PDMP insurance risk model. Acta Math. Appl. Sinica 19, 521-528.

WANG, G.-J, Zhang, C.-S AND WU, R. (2003b). Ruin theory for the risk process described by PDMPs. Acta Math. Appl. Sinica 19, 59-70.

YANG, H., ZhANG, Z. AND LAN, C. (2008). On the time value of absolute ruin for a multi-layer compound Poisson model under interest force. Statist. Prob. Lett. 78, 1835-1845.

YuAn, H. AND Hu, Y. (2008). Absolute ruin in the compound Poisson risk model with constant dividend barrier. Statist. Prob. Lett. 78, 2086-2094.

ZHANG, C. AND Wu, R. (1999). On the distribution of the surplus of the D-E model prior to and at ruin. Insurance Math. Econom. 24, 309-321.

ZHU, J. AND YANG, H. (2008). Estimates for the absolute ruin probability in the compound Poisson risk model with credit and debit interest. J. Appl. Prob. 45, 818-830. 